\title{
Quantitative 3D imaging of partially saturated granular materials under uniaxial compression
}

\author{
Marius Milatz $^{1}$ (D) $\cdot$ Nicole Hüsener $^{1} \cdot$ Edward Andò $^{2} \cdot$ Gioacchino Viggiani $^{2} \cdot$ Jürgen Grabe ${ }^{1}$
}

Received: 17 February 2021 / Accepted: 17 July 2021 / Published online: 23 August 2021

(c) The Author(s) 2021

\begin{abstract}
Gauging the mechanical effect of partial saturation in granular materials is experimentally challenging due to the very low suctions resulting from large pores. To this end, a uniaxial (zero radial stress) compression test may be preferable to a triaxial one where confining pressure and membrane effects may erase the contribution of this small suction; however, volume changes are challenging to measure. This work resolves this limitation by using X-ray imaging during in situ uniaxial compression tests on Hamburg Sand and glass beads at three different initial water contents, allowing a suctiondependent dilation to be brought to the light. The acquired tomography volumes also allow the development of air-water and solid-water interfacial areas, water clusters and local strain fields to be measured at the grain scale. These measurements are used to characterise pertinent micro-scale quantities during shearing and to relate them to the measured macroscopic response. The new and well-controlled data acquired during this experimental campaign are hopefully a useful contribution to the modelling efforts - to this end they are shared with the community.
\end{abstract}

Keywords Partially saturated granular soils $\cdot$ Shear strength $\cdot$ Uniaxial compression tests $\cdot$ X-ray computed tomography

\section{Introduction}

\subsection{Partially saturated soil behaviour}

It is well known that partially saturated soils behave differently to dry and water-saturated soils. The distribution of the air and water phases in the pore space, governed by capillarity, changes the hydraulic (e.g. decreasing permeability) and the mechanical (e.g. increasing shear strength and stiffness) properties of the granular assembly, as a function of degree of saturation. These macroscopic

This work is funded by the German Research Foundation (Deutsche Forschungsgemeinschaft, DFG) in the framework of Research Training Group GRK 2462: Processes in natural and technical Particle-Fluid-Systems [13] at Hamburg University of Technology (TUHH)

Marius Milatz

marius.milatz@tuhh.de

1 Institute of Geotechnical Engineering and Construction Management, Hamburg University of Technology (TUHH), Harburger Schloßstraße 20, 21079 Hamburg, Germany

2 Univ. Grenoble Alpes, Grenoble INP, CNRS, 3SR, 38000 Grenoble, France changes originate from the grain scale, as the result of two phases sharing the pore space, for example with menisci causing suction in the water phase. At the small scale, simple phenomena such as the ink-bottle effect can explain relatively complex behaviour at the macroscale such as the hysteretic nature of the water retention curve. The water retention curve (WRC) describes the relationship between gravimetric water content $w$ or degree of saturation $S_{\mathrm{r}}$ and matric suction $s$, more generally referred to as capillary pressure $p_{\mathrm{c}}$. The WRC governs the hydraulic behaviour of porous media during drainage and imbibition and can be measured on a macroscopic level by different laboratory experiments [14, 37].

Based on the early work of Terzaghi, the effective stress concept as a link between the hydraulic and mechanical stress state in soils was enhanced for the application to partially saturated soils by Bishop [8], adding the effect of suction.

\subsubsection{Imaging partially saturated soils}

$\mathrm{X}$-ray micro-tomography is a volumetric imaging technique that is now of sufficient spatial resolution to acquire images where sand grains, and air/water in pores can be 
distinguished. While CT scans were initially used to image stationary processes, more recent applications focus on a "4-dimensional" approach, where processes are captured over time with in situ imaging experiments. A review of different applications of CT in hydrology is given by [43] with a focus on flow in porous media. A recent overview of different CT-based techniques for the investigation of capillary-dominated fluid flow at the pore-scale is given by [33].

Regarding the specific application of CT to studying partially saturated soils, several authors have already applied CT to investigate the microscopic background of the macroscopic WRC [17, 20, 21, 24]. Other studies have investigated the hydro-mechanical behaviour of unsaturated soils: while [18, 20, 22, 23] investigated CT images obtained during triaxial compression of unsaturated sand, [9] and [30] used successive CT scans to investigate the socalled capillary collapse. This is a mechanical instability of the unsaturated grain skeleton occurring during imbibition due to a reduction of matric suction leading to a rearrangement of grains and macroscopic settlements.

CT data of good quality, i.e., with high resolution and low noise, obtained with the help of modern CT scanners using micro-focus X-ray tubes and high-resolution detectors, allow the investigation of microscopic properties and processes in granular media by means of image analysis. Beyond the present state and evolution of phase distributions, microscopic structures, such as the phase interfacial areas, can be measured and analysed [12]. The interfacial areas are believed to represent additional state variables that might help to better understand and model the effective stress state as well as shear strength in unsaturated soils [39]. Furthermore, the curvature of water menisci or the radii of curvature in unsaturated soils can be approximated and measured [5, 18], as well as contact angles [4].

Another area of focus is the study of the development of connected water or air phase clusters and connected grains inside and outside of the shear band in triaxial tests. Connected water clusters as well as grain contacts can be evaluated from microscopic CT data during a shearing process in order to investigate changes in the grain fabric as well as in the unsaturated state $[11,20,22,23,40]$. While $[22,23]$ evaluate the results of triaxial tests on unsaturated silica sand imaged with X-ray CT and find a decrease of pore water clusters in the shear zone with increasing axial strain, [20] shows that the volume of the largest water cluster is reduced upon triaxial shearing of unsaturated Hostun sand due to breakage of the water cluster into smaller volumes. This would mean that the number of water clusters is increasing during shearing in contradiction to the results found by $[22,23]$.

\subsubsection{Research questions}

This paper attempts to answer the following research questions:

(1) How does capillarity affect the macroscopic effective stress?

(2) What is the relationship between the microscopic capillary system, described by air-water distribution and degree of saturation, and measured shear strength and volumetric response on a macroscopic level?

(3) How do grain-scale quantities such as number and size of water clusters, location of capillary bridges and interfacial areas evolve during macroscopic shearing as grains rearrange?

For a better understanding of microscopic capillary effects and their link to the macro-mechanical behaviour, a miniature uniaxial compression device compatible with $\mathrm{X}$-ray scanning has been designed at Hamburg University of Technology [25].

As opposed to the triaxial compression tests already reported in literature, the low shear strengths measured in uniaxial compression (without lateral confinement) are expected to be strongly affected by capillarity, facilitating the study of the interplay between capillarity and shear strength as well as volumetric strain.

\section{Material and methods}

\subsection{Tested material}

In this work two different granular materials are investigated: "Hamburg Sand" (a coarse to medium coarse model sand, used in the soil laboratory at Hamburg University of Technology) and polydisperse glass beads (soda lime glass-SiLi beads type $\mathrm{S}$, manufactured by Sigmund Lindner GmbH, Germany). The grain size distribution of the glass beads approximates that of Hamburg Sand, so that the main difference is particle shape and dependent properties. Selected properties of the two granular materials are given in Table 1. The grain size distribution curves of both materials as well as photographs of unsaturated cylindrical specimens prior to shearing are shown in Fig. 1.

In order to assess the water retention behaviour of both investigated materials, the HYPROP evaporation test [31, 35], manufactured by UMS GmbH/METER Group, is used. The method allows the measurement of the primary drainage curves of cylindrical specimens with height 50 $\mathrm{mm}$ and diameter $80 \mathrm{~mm}$ during free evaporation from the top surface. With the help of suction measurements by 
Table 1 Main properties of the granular materials studied

\begin{tabular}{cccccc}
\hline \multicolumn{2}{l}{ Hamburg Sand } & & & & \\
\hline $\begin{array}{c}\rho_{s} \\
{\left[\mathrm{~g} / \mathrm{cm}^{3}\right]}\end{array}$ & $\begin{array}{c}e_{\min } \\
{[-]}\end{array}$ & $\begin{array}{c}e_{\max } \\
{[-]}\end{array}$ & $\begin{array}{c}d_{10} \\
{[\mathrm{~mm}]}\end{array}$ & $\begin{array}{c}d_{50} \\
{[\mathrm{~mm}]}\end{array}$ & $\begin{array}{c}d_{\max } \\
{[\mathrm{mm}]}\end{array}$ \\
\hline 2.64 & 0.52 & 0.805 & 0.45 & 0.68 & 2.0 \\
\hline Glass beads & & & & \\
\hline$\rho_{s}$ & $e_{\min }$ & $e_{\max }$ & $d_{10}$ & $d_{50}$ & $d_{\max }$ \\
{$\left[\mathrm{g} / \mathrm{cm}^{3}\right]$} & {$[-]$} & {$[-]$} & {$[\mathrm{mm}]$} & {$[\mathrm{mm}]$} & {$[\mathrm{mm}]$} \\
\hline 2.50 & 0.555 & 0.679 & 0.45 & 0.68 & 1.3 \\
\hline
\end{tabular}

$\rho_{s}$ : grain density, $e_{\min }:$ min. void ratio

$e_{\text {max }}:$ max. void ratio

$d_{10}$ : grain diameter at $10 \%$ passing

$d_{50}$ : grain diameter at $50 \%$ passing

$d_{\text {max }}:$ max. grain diameter

embedded tensiometer sensors, continuous primary drainage curves can be measured. Primary drainage curves for both tested materials are shown in Fig. 2 along with a curve fit using the Van Genuchten model [36] with parameters summarised in Table 2.

According to the measured primary drainage curves, both granular materials show very low capillary effects with air entry values below $1 \mathrm{kPa}$ matric suction. Slightly higher capillary effects are measured for the sand compared to the glass beads, which is further discussed in [27], where also hysteretic water retention data from cyclic drainage and imbibition experiments are presented.

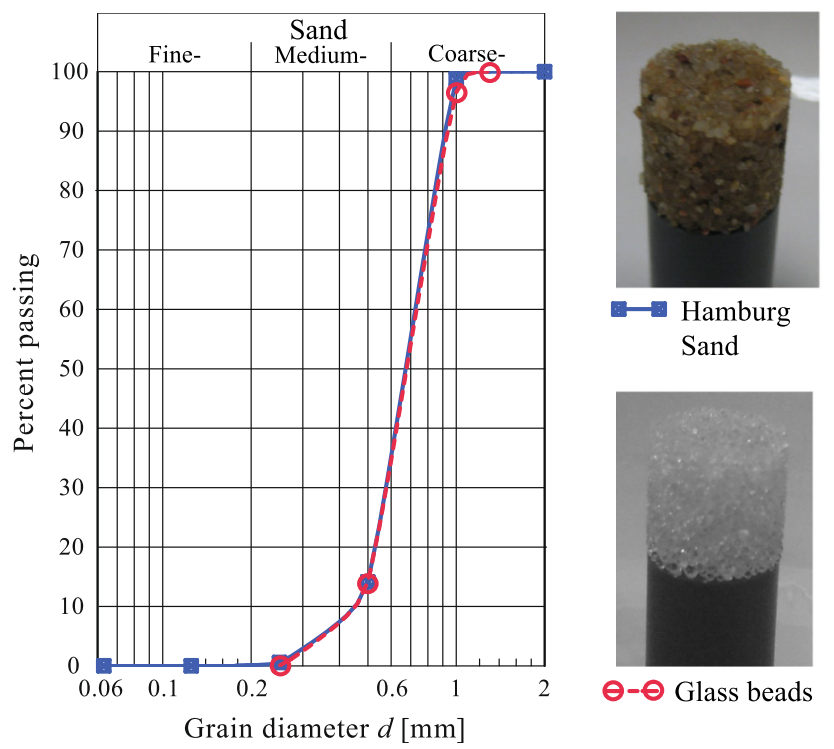

Fig. 1 Grain size distribution curves of Hamburg Sand and glass beads and photographs of free standing specimens of the different materials only kept together by capillary cohesion

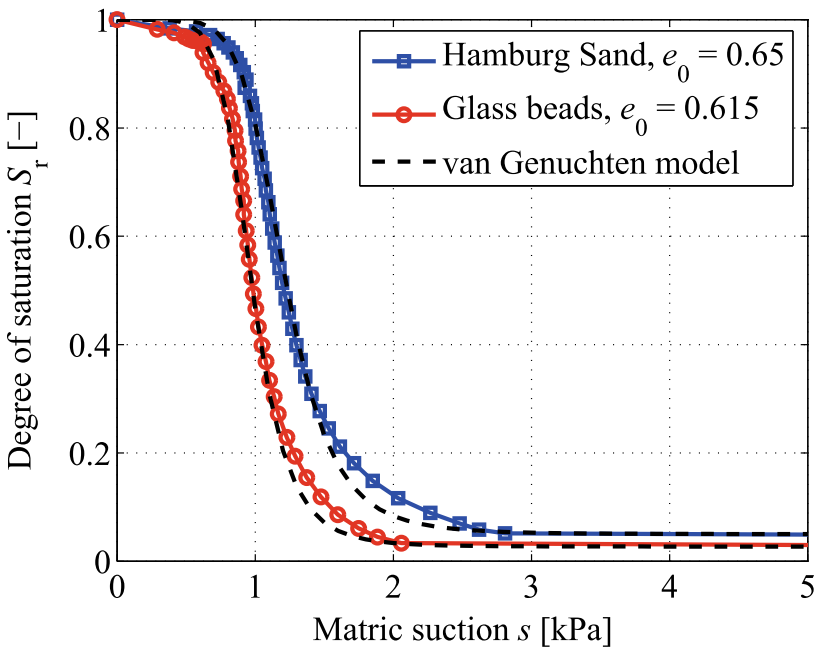

Fig. 2 Water retention curves of Hamburg Sand and glass beads as measured in the HYPROP evaporation test, curves fitted using the Van Genuchten model

Table 2 Parameters of the Van Genuchten model

\begin{tabular}{cccc}
\hline Material & $\alpha[1 / \mathrm{kPa}]$ & $n[-]$ & $S_{\text {res }}[-]$ \\
\hline Hamburg Sand & 0.847 & 7.3 & 0.050 \\
\hline Glass beads & 1.053 & 7.7 & 0.027 \\
\hline$\alpha, n,(m=1-1 / n)$ : fitting parameters $[36]$, \\
Sres residual degree of saturation
\end{tabular}

\subsection{Experimental set-up}

The miniature compression device developed by $[25,28]$ is used. The apparatus is controlled by a Raspberry Pi singleboard computer that drives a small stepper motor for axial loading of the specimen and records the load [26].

The apparatus is small enough to be placed on the rotation stage of a CT scanner and to allow for a small distance between X-ray source and specimen centre, see Fig. 3. A full view of the set-up in the scanning chamber is shown in Fig. 20 in the Appendix.

\subsubsection{Specimen preparation}

For the preparation of free standing soil columns (only kept together by capillary cohesion) with a height and diameter of $12 \mathrm{~mm}$, the specimen preparation method as shown in Fig. 21 in the Appendix is followed. The dry mass of granular material needed for the desired macroscopic void ratios in the target volume is weighed. The material is then mixed with the mass of de-ionised water needed to reach the chosen initial macroscopic water content. During this procedure, the dry material is firstly poured into a ceramic 

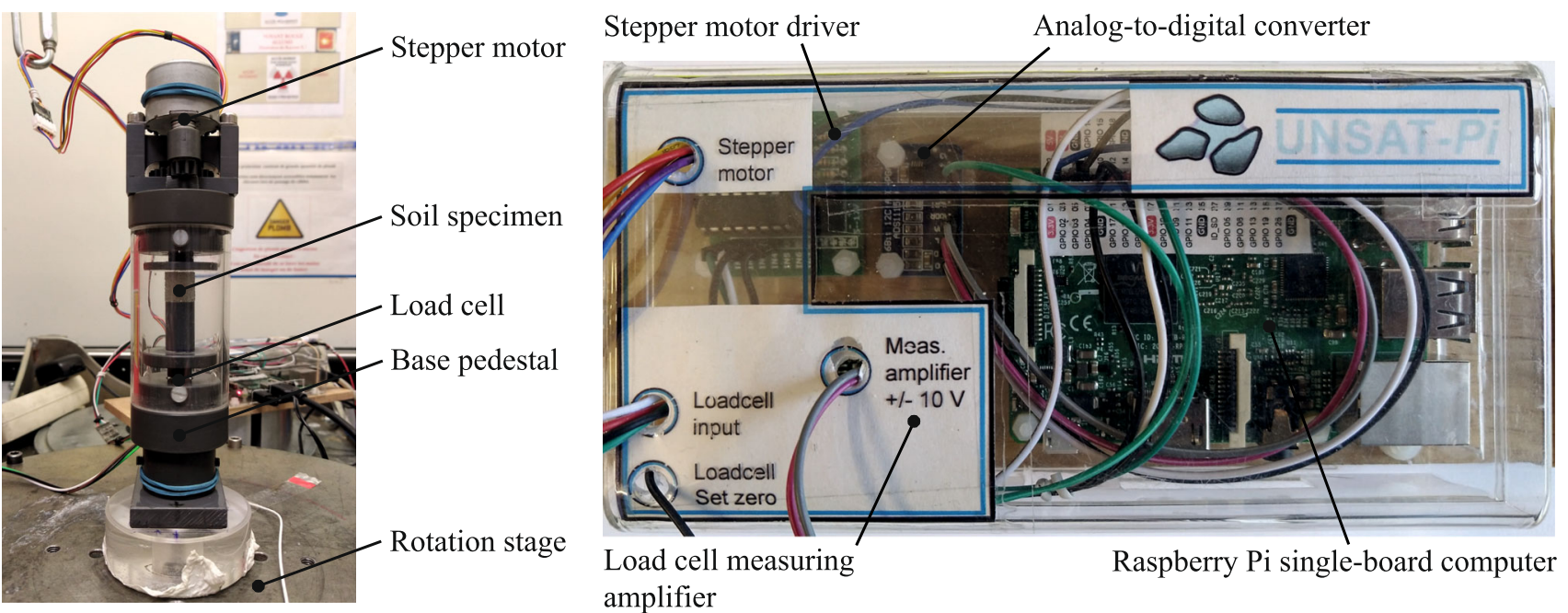

Fig. 3 Miniature uniaxial compression device placed on the rotation stage of the X-ray tomograph at Laboratoire 3SR (left) and UNSAT-Pi for test control with different hardware components (right)

cup, already containing the mass of water, and then the mixture is homogenised by careful mixing with a spoon. Finally, the mixture of granular material and water is filled into a hollow cylinder in layers, which are compacted using a piston. The surface of granular material is roughened with the tip of a screw driver in between each layer, in order to prevent the formation of artificial segregated layers. This is especially important in the case of the glass beads that tend to segregate. When the desired specimen height is reached by compaction in layers, the piston forming the specimen base pedestal is carefully pushed out of the hollow cylinder, thus leaving a free standing soil column.

With some practice, the specimen preparation procedure is achieved within ten to fifteen minutes without losing a single grain. For the monitoring of water content, which will deviate from the targeted macroscopic water content due to several sources of water loss, the specimen is weighed after installation in the uniaxial compression apparatus. Water loss may result from evaporation and from water adhesion to the ceramic cup as well as to the hollow cylinder. After enclosing the specimen under the loading piston inside the acrylic cell of the apparatus, evaporation will be slowed down but not stopped.

\subsubsection{Initial conditions of sand and glass bead specimens}

The initial water content of sand and glass bead specimens was varied to achieve initial macroscopic target degrees of saturation of $S_{\mathrm{r} 0}=0.3,0.5$ and 0.7 at an initial macroscopic void ratio $e_{0}=0.65$ for the sand specimens and $e_{0}=0.615$ for glass beads, corresponding to relative densities $D_{R}$ of 0.46 and 0.48 , respectively, meaning both are medium dense to dense. All initial specimen properties are summarised in Table 3, using the initial specimen height derived from CT data, which is more accurate than the measurement of specimen height with a ruler. The initial specimen height is calculated from the pixel size and the number of pixels between the top and bottom loading plates. The initial water content $w_{0}$ was determined by weighing the free standing specimens with a laboratory balance after preparation. Both initial water content and initial degree of saturation are therefore based on the true initial amount of water inside the specimen, net of the mentioned sources of water loss.

Table 3 Initial macroscopic specimen properties, note that height is measured from CT data

\begin{tabular}{|c|c|c|c|c|}
\hline \multicolumn{5}{|c|}{ Hamburg Sand } \\
\hline Test & $m_{\mathrm{d}}$ & $e_{0}$ & $w_{0}$ & $S_{\mathrm{r} 0}$ \\
\hline$[-]$ & {$[\mathrm{g}]$} & {$[-]$} & {$[-]$} & {$[-]$} \\
\hline 1 & 2.1710 & 0.6308 & 0.0508 & 0.2124 \\
\hline 2 & 2.1711 & 0.6383 & 0.1004 & 0.4153 \\
\hline 3 & 2.1717 & 0.6152 & 0.1468 & 0.6302 \\
\hline \multicolumn{5}{|c|}{ Glass beads } \\
\hline Test & $m_{\mathrm{d}}$ & $e_{0}$ & $w_{0}$ & $S_{\mathrm{r} 0}$ \\
\hline$[-]$ & {$[\mathrm{g}]$} & {$[-]$} & {$[-]$} & {$[-]$} \\
\hline 1 & 2.1013 & 0.5674 & 0.0738 & 0.3252 \\
\hline 2 & 2.1015 & 0.5673 & 0.1044 & 0.4601 \\
\hline 3 & 2.1015 & 0.5777 & 0.1374 & 0.5945 \\
\hline
\end{tabular}

$m_{\mathrm{d}}:$ dry mass 


\subsubsection{Uniaxial compression tests with CT scanning}

Uniaxial compression tests are performed "in situ" inside the X-ray tomograph at Laboratoire 3SR [38], as shown in Fig. 20 in the Appendix. For each test, loading is interrupted at a number of points to allow CT scanning.

Every test series starts with a careful docking procedure to ensure the contact of the loading plate with the specimen top, after which an initial CT scan (at zero axial strain) is acquired. The scan settings are summarised in Table 4 resulting in a $3 \mathrm{D}$ volume representing the $\mathrm{CT}$ value (roughly X-ray attenuation) of the whole specimen volume and parts of the bottom and top loading plates at a voxel size of $11 \mu \mathrm{m} / \mathrm{px}$ with an acquisition time of approx. 36 min. In order to minimise specimen disturbance due to acceleration effects, the rotation stage is moved continuously during image acquisition with a low rotation rate of approximately 10 degrees/minute. Abrupt changes in rotation speed are avoided with the help of smooth acceleration or deceleration controls.

The displacement increment applied in every loading step was equal to $\Delta s=0.3 \mathrm{~mm}$ in most cases, corresponding to an axial strain increment of $\Delta \varepsilon=0.025$ at a target specimen height of $12 \mathrm{~mm}$ after specimen preparation. However, for a finer strain resolution, in one of three test series on sand and glass beads (test 3 ) a displacement increment of $\Delta s=0.12 \mathrm{~mm}$ was applied, resulting in an axial strain increment of $\Delta \varepsilon=0.01$. The specimens were sheared to a target maximum macroscopic strain of $\varepsilon_{\max }=0.15$, corresponding to an axial displacement of $1.8 \mathrm{~mm}$.

Regarding the measurement of axial force, the load cell data were recorded continuously during axial loading steps and during CT scans and was cleaned from obvious data jumps and measurement drifts during CT scanning.

\subsection{Pre-processing of CT data}

CT scans are reconstructed using X-Act by RX Solutions resulting in a 16-bit-greyscale volume. Afterwards, these raw data are further processed using the open source software SPAM [34] and the commercial image processing

Table 4 Settings of the CT scans

\begin{tabular}{ll}
\hline X-ray tube voltage & $120 \mathrm{kV}$ \\
X-ray tube current & $82 \mu \mathrm{A}$ \\
Projections $/ 360^{\circ}$ revolution & 1200 \\
Isotropic voxel size & $11 \mu \mathrm{m}$ \\
Averaging & 4 \\
Acquisition time per scan & $\approx 0.6 \mathrm{~h}$ \\
Acquisition mode & Continuous rotation \\
\hline
\end{tabular}

and analysis software Avizo by Thermo Fisher Scientific. The image processing consists of the following steps:

1. Histogram normalisation: a linear rescaling of all greylevels is performed in order to ensure that the three phases of interest (air, water, grain) have the same mean greylevel in all scans.

2. Filtering: A 3D median filter is applied, followed by a non-local means filter [15] as shown in Fig. 4.

3. Phase identification: A step-wise semi-automatic approach [19] is used: markers are computed from the gradient of the image, which are used in a markersbased watershed algorithm, which should minimise the phase identification errors for partial-volume voxels between grain and air.

\subsection{Global measurements from CT data}

With the phase identification carried out for each acquired volume, we study the evolution of the specimen height, specimen volume (normally not measured in a uniaxial compression test) and pore water volume.

The volumetric strain $\varepsilon_{\mathrm{v}}$ is especially interesting because it allows the interpretation of the volumetric shear behaviour of unsaturated granular assemblies, i.e., the tendency towards contractancy or dilatancy, at quasi-zero confining pressure. The volume of the sample is measured in every scan by first dilating and then eroding the non-air phases until the internal voids are filled. While the dilation algorithm in Avizo adds voxels to all solid surfaces, thus filling inner pores, the erosion algorithm removes them again to restore the outer specimen surfaces. Similar procedures have been proposed by [1] and [20] for measuring the bulk

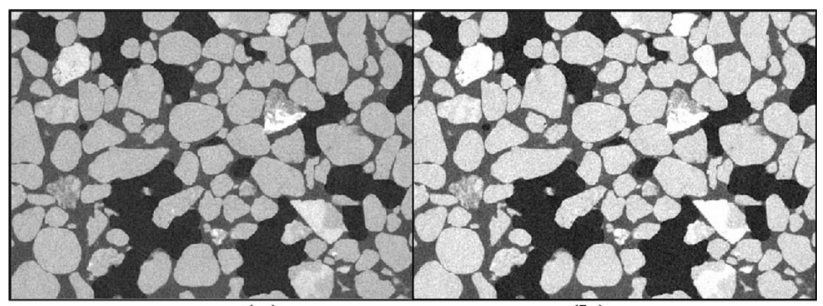

(a)

(b)

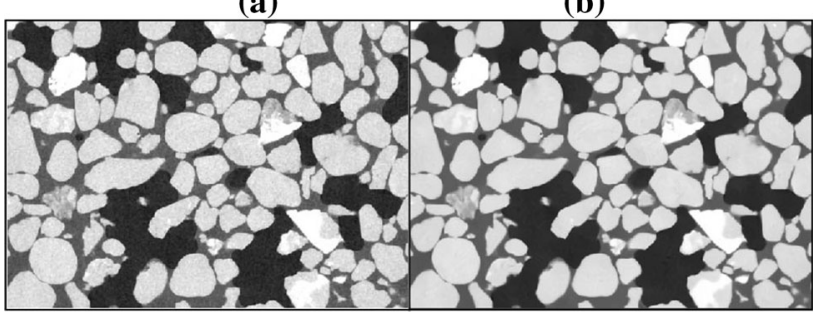

(c)

(d)

Fig. 4 Steps of image improvement by different operations:a Raw image data,b greyscale normalisation, c median filter,d non-local means filter 
volume of a specimen in a triaxial test and by [45] to analyse the intra-particle pores in carbonate sands. The pores at the boundaries of the sample (top, bottom and lateral boundaries) are also filled by this morphological image processing operation, leaving a surface resembling to a smooth membrane strained over a granular specimen. Figure 22 in the Appendix shows the results of this filling procedure for one scan, comparing a photograph of the specimen to voxel-based CT data of the non-air phases and to the final specimen volume with filled pores. The total volume of the specimen is thus a voxel count of the nonpore phase; tracking this quantity through time gives $\varepsilon_{v}=\Delta V / V_{0}$, with $\Delta V$ and $V_{0}$ being the volume change and the initial specimen volume, respectively.

The cross-sectional area $A$ of the specimen can also be directly computed for each scan from the filled image, allowing the calculation of the axial stress from the measured axial force.

Since the experiments take several hours, evaporation cannot be neglected, because it directly affects pore water volume. Although measures are taken to minimise evaporation (i.e., the specimen is placed in a closed acrylic cell, where no outside air movement can affect the specimen), evaporation cannot be totally avoided. To quantify the effect of evaporation, the water content of the specimens is monitored by initial weighing and by measurement of the final water content at the end of the experiments. Looking

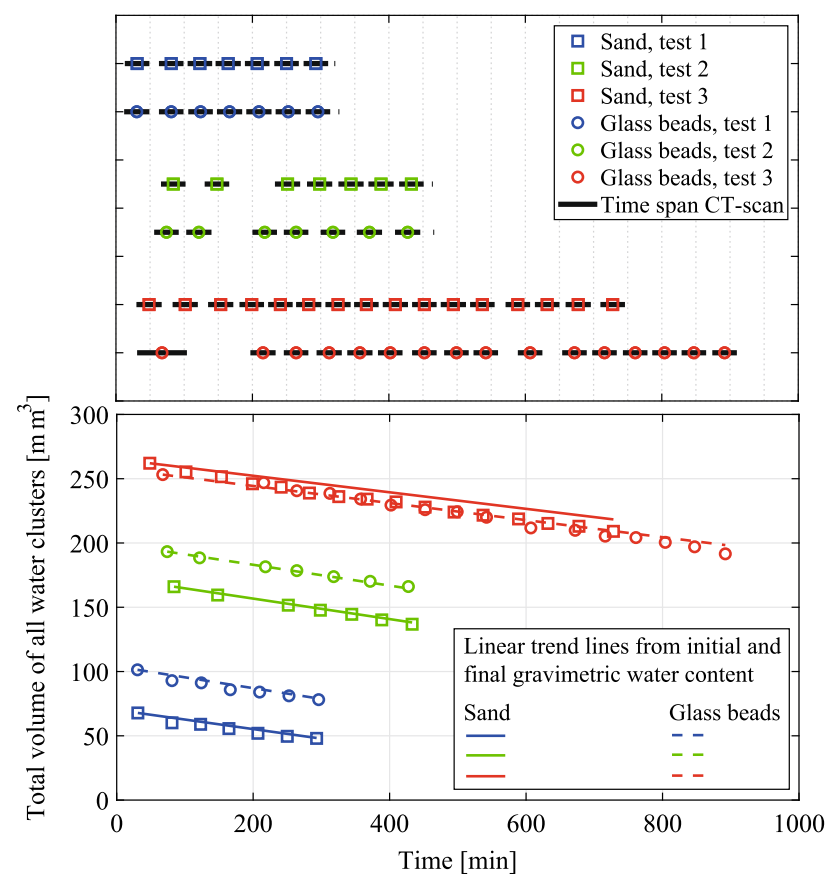

Fig. 5 Timing of all CT scans during uniaxial compression (top) and development of pore water volume during the whole experiment based on voxel data and compared to trend lines derived from initial and final gravimetric water content (bottom) now into the measured water contents in the scans, Fig. 5 (top) shows the scan times and scan duration for all experiments, with $t=0$ corresponding to first weighing after specimen preparation. The top plot is to be compared to the bottom plot, which shows the water volume (computed in a subvolume with an initial height of 970 voxels i.e., $10.67 \mathrm{~mm}$ that tracks the material during compression) with time. Straight trend lines calculated from initial and final gravimetric water contents are also plotted in this space, indicating that an essentially linear loss of water with time is a reasonable approximation. In addition to the effect of evaporation, a change in specimen water content due to a possible outflow of pore water at the top and bottom loading platens cannot be excluded. This outflow might occur with dilating pores at elevated degrees of saturation and should be investigated in further studies. Flow at the boundaries is not evaluated in the present paper, which mainly focuses on central subvolume-based data, excluding the specimen boundaries due to image noise and a resulting inaccurate segmentation of the water phase in the corresponding local data at the boundaries.

\subsection{Local measurements from CT data}

Starting again from the trinarised images, local statistics of voxel counts (yielding measurements of $e$ and $S_{\mathrm{r}}$, for example) are obtained with the definition of a measurement subvolume. The subvolumes used have fixed edge lengths and are centred inside the specimen in each scan (i.e., they track the displacement of the specimen's centre due to shortening but don't change size). Figure 23 in the Appendix shows the vertical homogeneity of these measurements. All subvolumes, their locations and dimensions, used here for the calculation of local microscopic specimen properties, are summarised in Table 5.

Local voxel counting can be complemented by more advanced measurements such as the calculation of the interfacial areas $a^{\text {nw }}$ (air-water interfacial area) and $a^{\mathrm{sw}}$

Table 5 Subvolumes for the calculation of local microscopic properties

\begin{tabular}{ll}
\hline \multicolumn{2}{l}{ Volume averaged properties } \\
\hline Fix dimensions $\left[\mathrm{px}^{3}, \mathrm{~mm}^{3}\right]$ & Calculated properties \\
$600 \times 600 \times 600,6.6 \times 6.6 \times 6.6$ & $e, S_{\mathrm{r}}$ \\
\hline \multicolumn{2}{l}{ Volume averaged properties per slice } \\
\hline Fix dimensions $\left[\mathrm{px}^{3}, \mathrm{~mm}^{3}\right]$ & Calculated properties \\
$600 \times 600 \times 900,6.6 \times 6.6 \times 9.9$ & $e, S_{\mathrm{r}}$ \\
\hline Interfacial areas & \\
\hline Fix dimensions $\left[\mathrm{px}^{3}, \mathrm{~mm}^{3}\right]$ & Calculated properties \\
$300 \times 300 \times 300,3.3 \times 3.3 \times 3.3$ & $a^{\mathrm{nw}}, a^{\mathrm{sw}}$ \\
\hline Water clusters & \\
\hline Initial height $[\mathrm{px}, \mathrm{mm}]$ & Calculated properties \\
$970,10.67$ & Cluster volume statistics \\
\hline
\end{tabular}


(a)

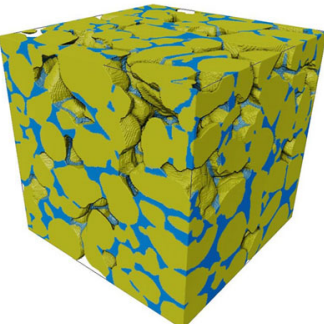

(b)

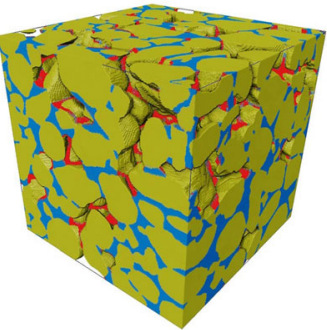

(c)

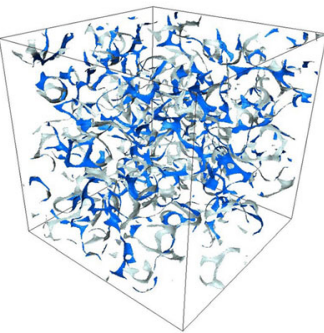

(d)

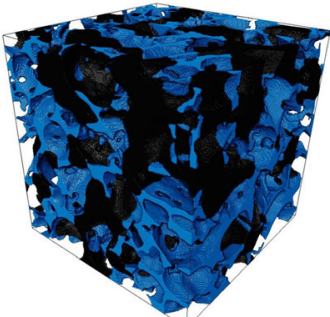

(e)

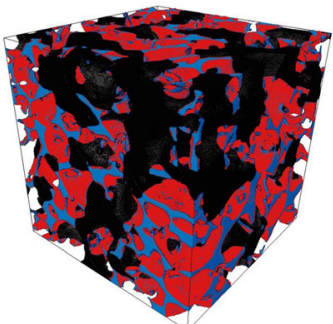

(f)

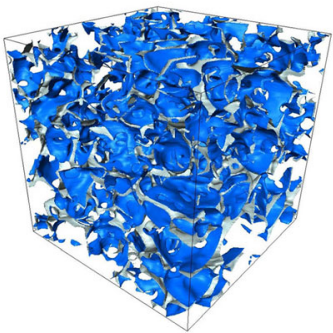

Fig. 6 Extraction of interfacial areas for a sand specimen: a Subvolume with sand phase (yellow) and water phase (blue), b Sand and water phase with $a^{\mathrm{nw}}$ highlighted in red, $\mathbf{c}$ Extracted $a^{\mathrm{nw}}$ rendered as a surface, d Subvolume with water phase (blue) and air phase (black), e Water and air phase with $a^{\mathrm{sw}}$ highlighted in red, f Extracted $a^{\mathrm{sw}}$ rendered as a surface

(solid-water or grain-water interfacial area). The air-water interfacial area $a^{\mathrm{nw}}$ as well as the grain-water interfacial area $a^{\mathrm{sw}}$ are calculated as the boundaries of the respective couple of phases, discretised by a surface mesh of triangles. The extraction of $a^{\mathrm{nw}}$ and $a^{\mathrm{sw}}$ from a subvolume of $300 \times 300 \times 300$ px is illustrated in Fig. 6 .

Furthermore, individual water clusters can be identified and individually studied for their volume, location, etc. in a representative centred initial specimen height of 970 voxels $(10.67 \mathrm{~mm})$. This subvolume has been selected to avoid image noise due to boundary effects close to the top and bottom loading plates, while at the same time capturing most of the pore water inside the specimens. The height of this subvolume is adapted to account for axial compression of the specimen. Individual water clusters are defined as contiguous patches of connected water voxels, which are numbered in the form of a labelled image, allowing properties of each cluster to be measured. Here, two voxels with at least one common vertex are assumed to be connected. Figure 7 is an example of a 3D rendering of labelled water clusters with one colour per cluster.

\subsection{Discrete (granular) analysis}

In the images acquired there is sufficient spatial resolution to identify individual grains, which offers a convenient basis for the description of the granular samples studied. Individual grains are defined in a voxelised image by $l a$ belling the voxels of the solid phase, such that all voxels belonging to a specific particle are given a unique number (label)-here the ITK watershed [7] is used with some post-processing.

Given particle centres and sizes, a Radical Delaunay (or Laguerre) triangulation is computed, linking neighbouring particle centres with tetrahedra. This spatial discretisation is convenient for defining individual pores between particles [10] and thus their degree of saturation. Individual pores are simply all the non-solid voxels enclosed in a tetrahedron, as identified from the segmented images.

On top of offering a convenient way to define pores, access to individual particles opens the door to the measurement of their kinematics between two imaged states (i.e., during loading). Here, the "discrete DIC" script in the open-source software SPAM [34] (implementing grain-based image tracking from [16]) is used. Particles are tracked based on their greyscale image by extracting the image in the labelled (reference) image, and solving a classical image correlation problem to minimise a greyscale residual in the deformed configuration. In this case the non-rigid correlation engine in SPAM is used to solve the image correlation problem, yielding a 3D displacement and homogeneous deformation matrix for each labelled particle.

Combining grain kinematics from discrete DIC and the triangulation mentioned above, a strain tensor is computed $[6,44]$ for each tetrahedron (which is relatively noisy since displacement gradients are computed only with 4 displacements). Grain displacements and rotations, or strain invariant fields can be visualised, e.g. [3], and can be of interest in themselves. The focus of analysis here will be on

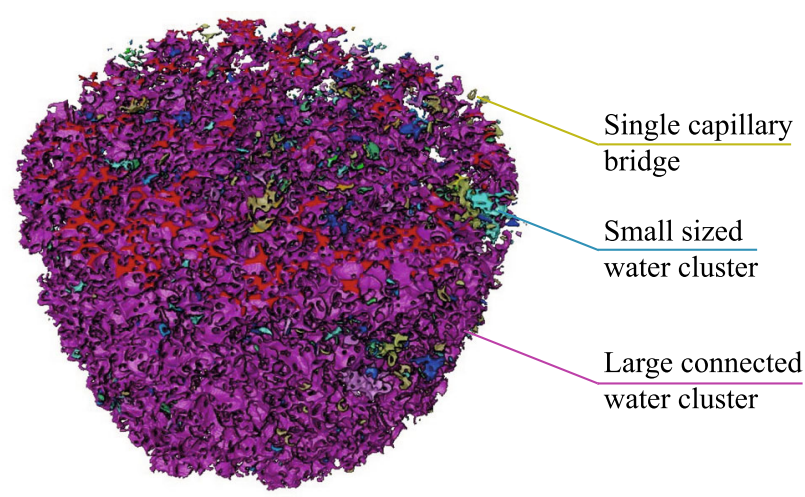

Fig. 7 3D rendering of water clusters for a sand specimen after 6 shear steps 
the coupling between pore saturation and the local strain computed around those pores.

\section{Experimental results}

\subsection{Macroscopic sample response}

The stress-strain curves for the different specimens tested are shown in Fig. 8, combining axial force measurements and sample cross-sectional areas (from CT) to compute axial stresses and sample volumes (also from CT) to derive volumetric strains (Fig. 24 in the Appendix shows a 3D rendering of the solid and water volumes for one test to illustrate the sample behaviour). The axial stresses-and therefore the shear strengths-reached in the sand samples are significantly higher than in the glass beads. During CT scans-with loading halted-the axial stress reduces in time, possibly due to relaxation; this is more noticeable in the sand samples. The peak axial stress does not seem to depend strongly on the initial degree of saturation unlike the monotonic loading tests reported in [25]. This observation might be a consequence of small specimen sizes in combination with spatial variations of density and degree of saturation. Furthermore, the overall lower peak stresses
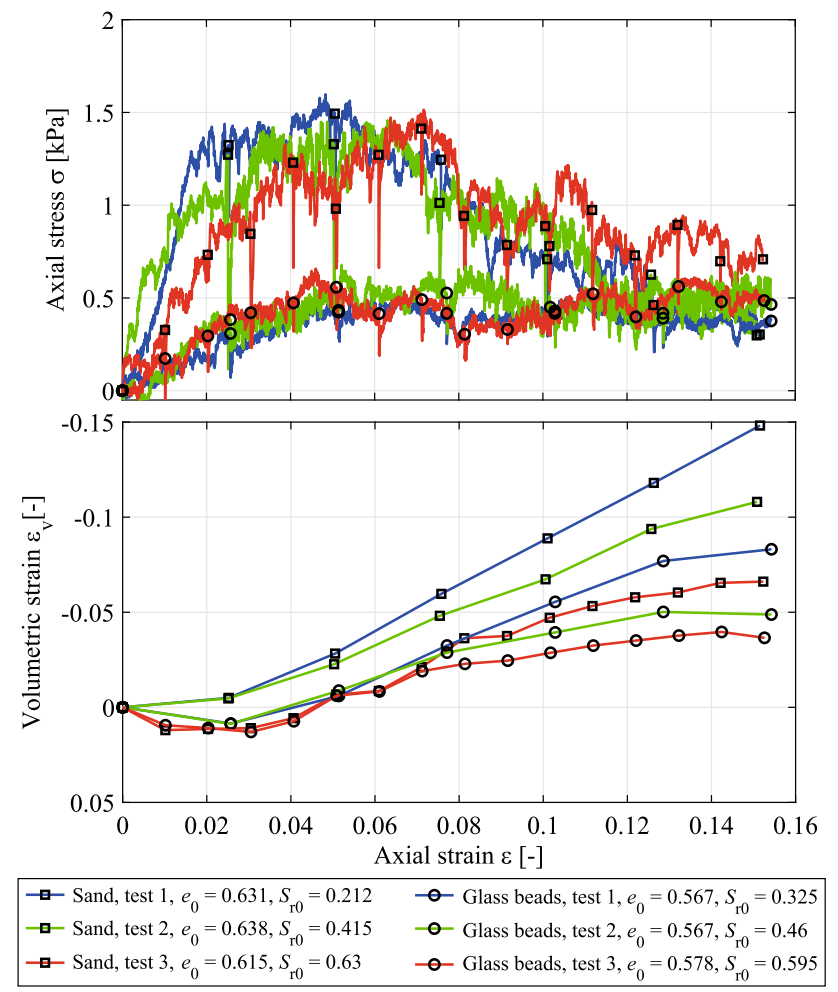

Fig. 8 Results of uniaxial compression on Hamburg Sand and glass beads for different initial macroscopic degrees of saturation $S_{\mathrm{r} 0}$ : Axial stress (top) and volumetric strain (bottom) vs. axial strain obtained in this work might also be explained by numerous stops with stress relaxation during loading.

Figure 8 shows that all specimens dilate after $3 \%$ shortening, with the two drier sand specimens dilating from the very beginning. At equivalent water content, the sand specimens dilate more. Unlike the axial stress peak, the volume changes clearly depend on the initial degree of saturation with the drier specimens dilating more.

\subsubsection{Evolution of vertical profiles of void ratio and degree of saturation}

A 3D subvolume entirely contained inside the specimen is defined (Fig. 23 in the Appendix), allowing the calculation of $e$ and $S_{\mathrm{r}}$ within some gauge volume. Figures 9 and 10 show these horizontally averaged quantities evolving for tests on Hamburg Sand and glass beads, respectively.

In the plots of void ratio evolution for both materials, the horizontally averaged measurement for each time step (evolving colours) is complemented by a trend line for the initial state, as well as three vertical lines representing the standard minimum and maximum void ratios as well as the measured initial value of macroscopic void ratio. The average value of the initial void ratio corresponds well to the measured one, especially for the sand specimens. The trend of the initial vertical distribution of void ratio reveals in most cases a very slight increase with height, indicating-even on $12 \mathrm{~mm}$ high specimens-a slight effect of compression of lower layers during specimen preparation. In all cases for both materials, the initial vertical void ratio distribution is essentially bounded by $e_{\min }$ and $e_{\max }$, but as the specimens dilate everywhere and monotonically during compression, the local value of $e_{\max }$ is significantly exceeded. Note that $e_{\min }$ and $e_{\max }$ are conventional values measured on dry material; much looser arrangements are possible with the small contribution of capillary forces-indeed this shearing process thus appears to push the material into this looser-than- $e_{\max }$ state. Possibly due to friction with the end-platens, in a number of cases the most significant dilation appears to be in the middle of the subvolumes-since the subvolumes have a fixed height of $900 \mathrm{px}$, these boundary effects close to the top and bottom loading plates occur with increasing loading. Regarding the water phase, it must be noted that the $\mathrm{CT}$ data contain noise and other artefacts in the vicinity of the bottom and top loading plates, which are responsible for the high gradients and values of degree of saturation, when these zones enter the field of view in Figs. 9 and 10 for advanced loading steps.

The plots of the evolution of degree of saturation $S_{\mathrm{r}}$ for both materials are also complemented by a trend line for the initial state, as well as a vertical line representing the 

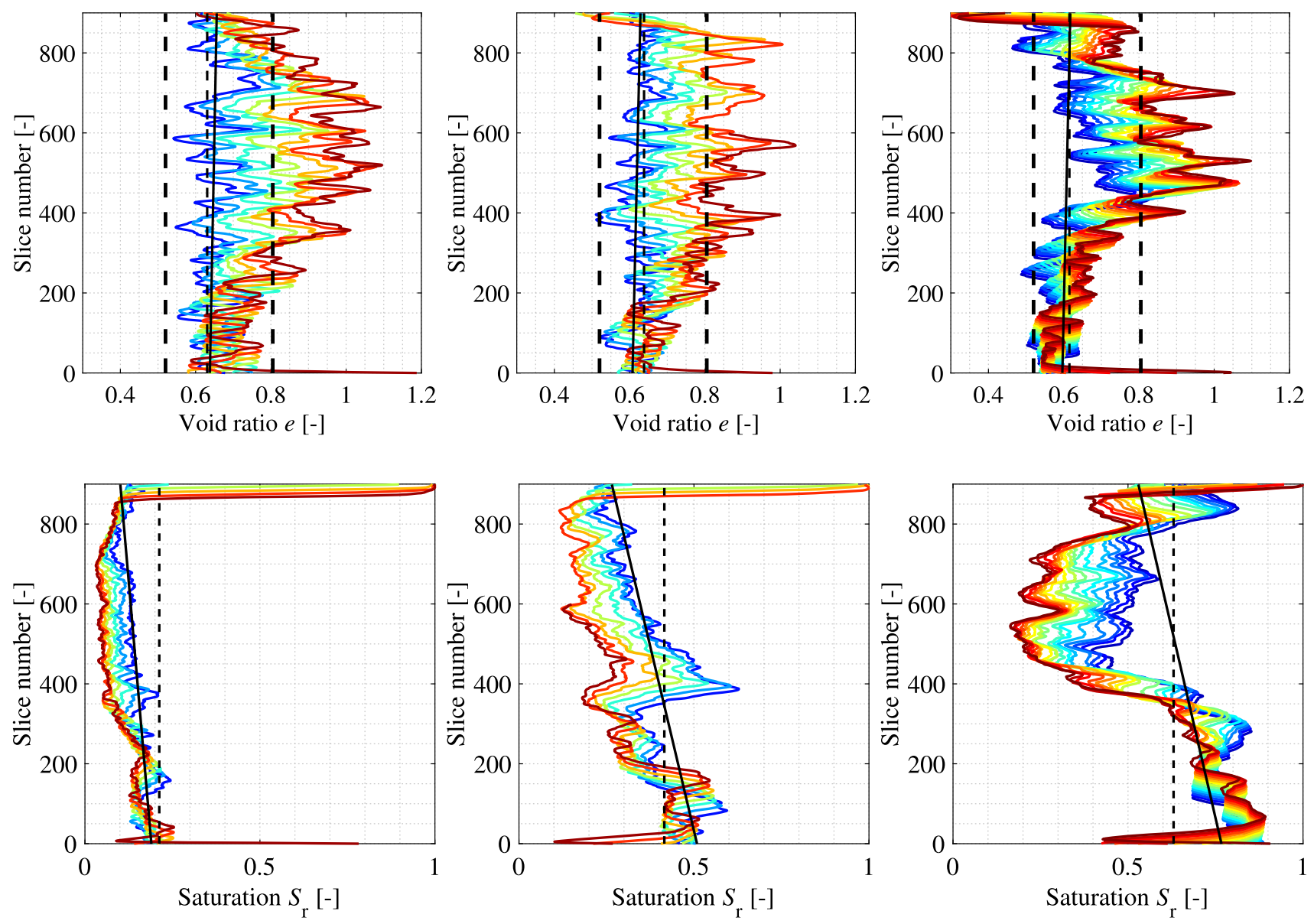

Test 1

Test 2

Test 3
- - Bounds given by $e_{\min }$ and $e_{\max }$
- - Initial macroscopic $e_{0}$ or $S_{\mathrm{r} 0}$
- Trend line of initial state of $e_{0}$ or $S_{\mathrm{r} 0}$ derived from subvolume-CT data

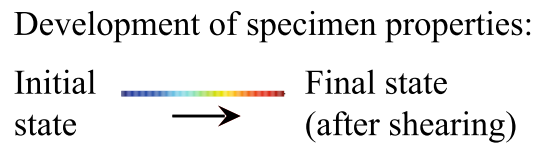

Fig. 9 Slice-wise specimen properties derived from a subvolume of $600 \times 600 \times 900 \mathrm{px}$ for Hamburg Sand

initial value of macroscopic degree of saturation. The initial macroscopic $S_{\mathrm{r}}$ appears to overestimate what is measured in the drier samples. $S_{\mathrm{r}}$ increases from top to bottom for all specimens (likely due to gravity even on this scale), the effect being larger for higher initial degrees of saturation and generally more pronounced in the glass bead specimens due to their lower water retention compared to the sand.

Comparing the evolution of $S_{\mathrm{r}}$ and $e$, it appears that the elevations that dilate the most are those with the lower degree of saturation - this is especially visible in the wetter specimens.

Trends for both $S_{\mathrm{r}}$ and $e$ computed on average on a smaller averaging volume chosen to avoid boundary effects are presented and briefly discussed in the Appendix in Figs. 25 and 26, the latter figure including a correction for evaporation. These results confirm the previous findings and also allow insight into changes of degree of saturation only due to volume change (without the effect of evaporation). Furthermore, differences between macroscopic and local void ratio and degree of saturation are discussed in the Appendix around Table 6.

\subsubsection{Evolution of interfacial areas}

The development of the air-water interfacial area $a^{\text {nw }}$ and the solid-water interfacial area $a^{\mathrm{sw}}$ versus axial strain is shown in Fig. 11 for all experiments on Hamburg Sand and glass beads. The interfacial areas are calculated for a subvolume of $300 \times 300 \times 300$ px $(3.3 \times 3.3 \times 3.3 \mathrm{~mm})$.

The solid-water interfacial areas monotonically decrease during shearing for all tests on both materials. The 

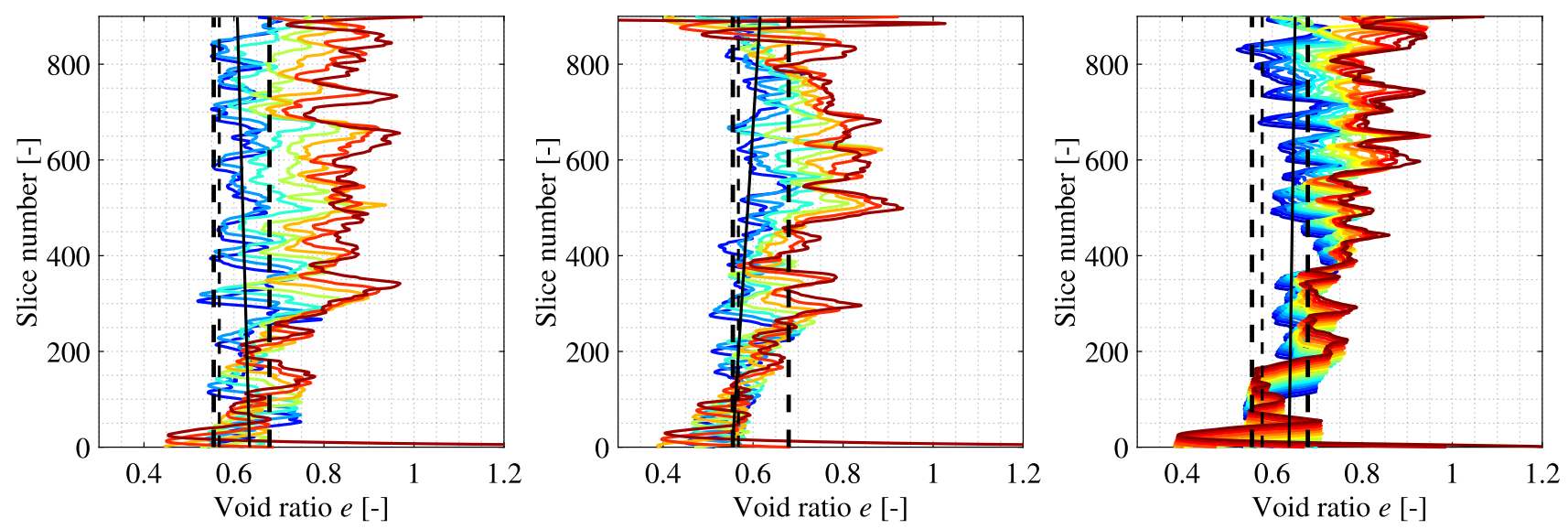

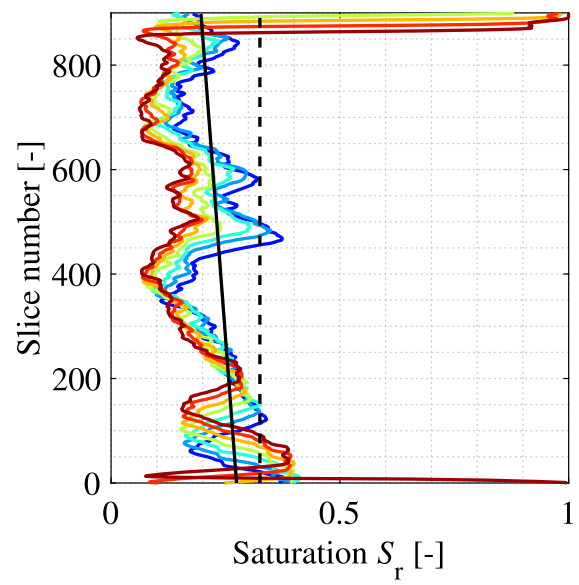

Test 1

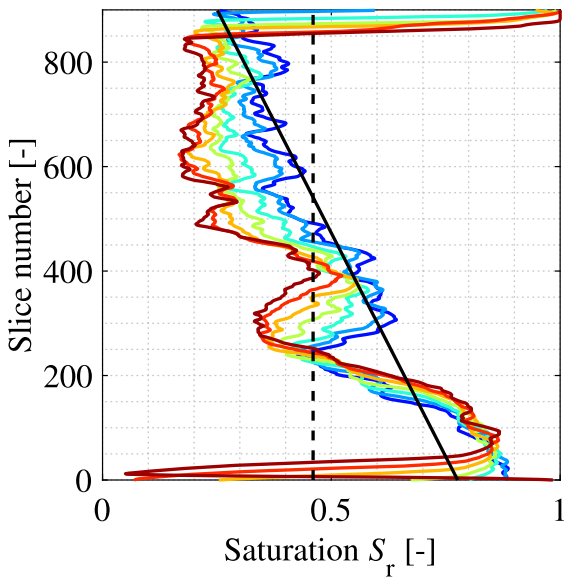

Test 2

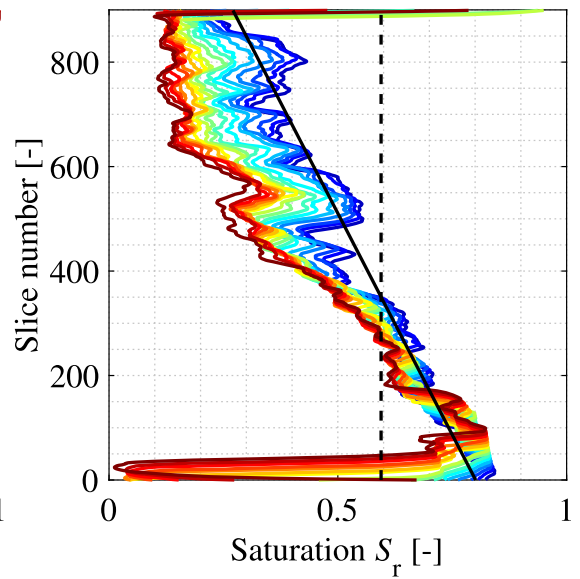

Test 3
- - Bounds given by $e_{\min }$ and $e_{\max }$
- - Initial macroscopic $e_{0}$ or $S_{\mathrm{r} 0}$
- Trend line of initial state of $e_{0}$ or $S_{\mathrm{r} 0}$ derived from subvolume-CT data

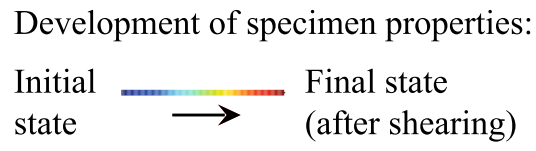

Fig. 10 Slice-wise specimen properties derived from a subvolume of $600 \times 600 \times 900 \mathrm{px}$ for glass beads

air-water interfacial areas for both materials, however, appear to evolve differently depending on the initial degree of saturation, decreasing during shearing for the driest specimens, increasing and plateauing for the intermediate ones (that start from the highest initial values), and increasing, peaking and eventually starting to decrease for the wettest specimens.

The observed changes in interfacial areas as well as their different initial values can be explained by the changes in degree of saturation during the experiments (which include the uncontrolled effect of evaporation), shown in Fig. 12. The limits on the value of $a^{\text {sw }}$ are expected to be the specific grain surface area minus the grain contact areas per unit volume for full saturation, and $a^{\mathrm{sw}}=0$ for $S_{\mathrm{r}}=0$. The trend for $a^{\mathrm{sw}}$ reveals a monotonic decrease of interfacial area with decreasing degree of saturation-and thus available water, which makes sense.

The limits of the air-water interfacial areas are more difficult to establish a priori: since these will depend strongly on the curvature of the air-water interface (and thus will tend to track suction rather than degree of saturation) which will depend on the pore size (and thus grain size) distribution, as well as the spatial distribution of water in the medium. Studying Figure 12, $a^{\text {nw }}$ shows a maximum in between $S_{\mathrm{r}}=0$ and $S_{\mathrm{r}}=1$ for both materials; the sand data (square symbols) show the intermediate sample reaching the highest air-water interfacial area at $S_{\mathrm{r}} \approx 0.25$. For the glass beads (circular symbols), the maximum value of $a^{\mathrm{nw}}$ is reached again for the intermediate sample, but at a significantly different $S_{\mathrm{r}} \approx 0.4$. Interestingly, a very similar trend is reported by [39] for a packing of glass beads with a 
narrow grain size distribution and grain diameters between 0.2 and $0.4 \mathrm{~mm}$.

\subsubsection{Evolution of water clusters}

The evolution of the interfacial areas discussed above might be better understood by considering the distribution of water in the sample as a series of discrete patches of water, and their evolution with shearing. Water clusters are identified and numbered in each X-ray scan, and the volume of each cluster is computed. Different domains of cluster volume are identified:

- Domain 0 (smallest clusters): broken pendular rings (and possibly some image noise)

- Domain 1: visible intact and broken pendular capillary bridges

- Domain 2: assemblies of capillary bridges

- Domain 3: water clusters up to the second largest water volume

- Domain 4: the largest single water cluster inside a specimen

For every domain, the water clusters included in the specimen for each scan have been counted in order to evaluate the data in the form of a series of histograms, shown in Fig. 13 for the driest sample of both Hamburg Sand (top) and glass beads (bottom) for different scans (with "shear step" 1 indicating the first scan). The histogram shows the development of water cluster count for ten volume bins. Figure 13 also shows 3D renderings of domains $0-4$ (with different colours indicating different numbered water clusters) for the first scan to illustrate their morphology. It is important to note that domain 4 is not in the histogram since it contains only a single cluster, and the count for domain 0 is not shown because it includes many thousands of clusters.

For the sand specimen, the histogram shows an increase of the number of water clusters throughout the test. Although evaporation is also reducing the overall pore water volume during the whole experiment, larger water clusters seem to break down into smaller clusters during shearing. Therefore, a trend towards more numerous small water clusters for all considered volume domains can be observed (likely at the expense of the overall volume of the largest cluster). On a smaller scale, shearing seems to cause a progressive separation of water clusters and also of smaller capillary bonds, which leads to a change in capillarity. This process becomes very obvious when the different domains of water volumes are visualised during shearing, as shown in Fig. 14. In this figure, every independent water cluster is marked with a different colour. As the colours remain the same in every column, it can be noticed that all assembled water clusters per step shown in

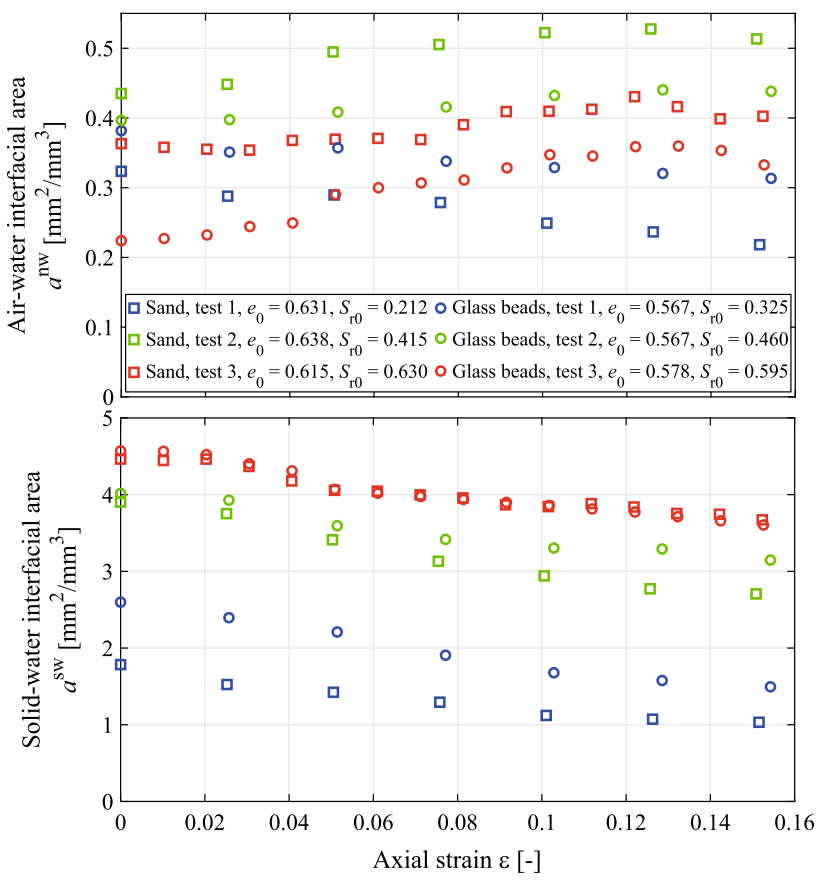

Fig. 11 Development of air-water interfacial area (top) and solidwater interfacial area (bottom) in a centred subvolume of $300 \times 300 \times 300$ px during uniaxial compression of the sand and glass bead specimens

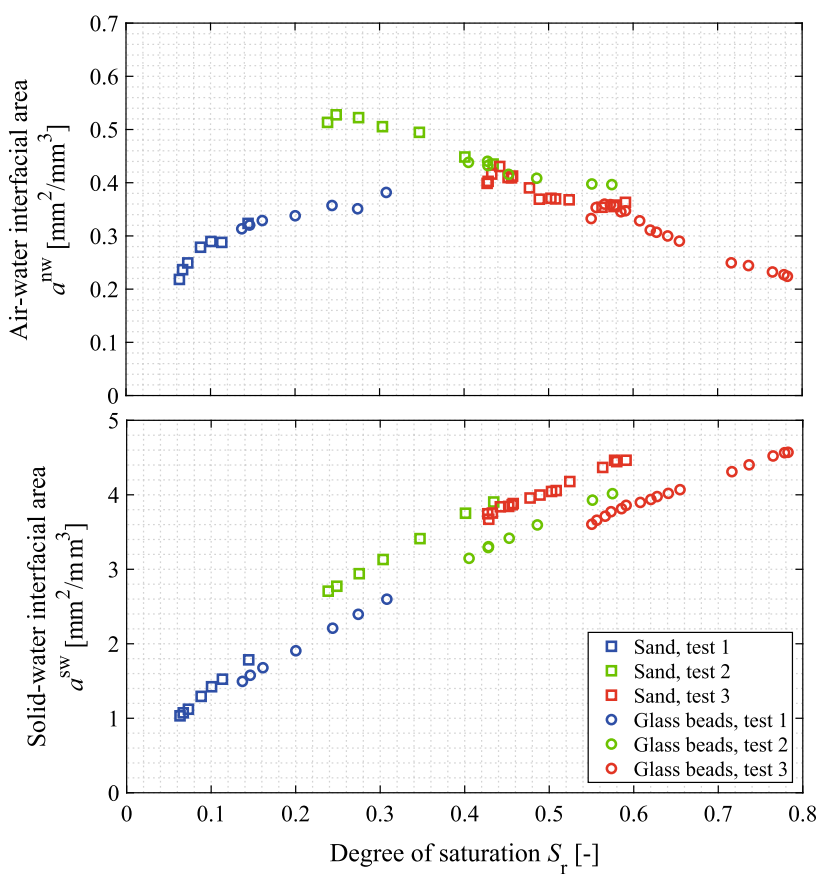

Fig. 12 Development of air-water interfacial area (top) and solidwater interfacial area (bottom) versus local degree of saturation in a centred subvolume of $300 \times 300 \times 300$ px during uniaxial compression of the sand and glass bead specimens 
row (a) are made up by finer clusters of all considered domains in rows (b) to (f).

A pronounced multiplication of smaller water clusters in domains 0 and 1 can be seen, which might also be due to evaporation. However, especially the development of larger water clusters of domains 3 and 4 shows that the larger clusters are torn apart with the dilating grain skeleton and thus separate into smaller clusters. A more detailed look at domain 1 reveals many single ring-shaped capillary bonds between two individual sand grains, many of which remain intact during shearing.

Comparing the histogram and visualisations of the discussed sand specimen to the glass beads (Figs. 13 and 15), generally, fewer water clusters exist in the selected volume domains (note the different vertical scale axis on the histograms). As with the sand, also in glass beads smaller water clusters are created during shearing, but they are relatively fewer compared to the total number of clusters. While domain 1 includes many small capillary bridges appearing as rather ideal rings that increase in number after some initial oscillations of value and remain intact during most shear steps, larger clusters, especially from the domain 3, multiply due to the breaking up of larger clusters. In contrast to the sand specimen, the single largest water cluster contained in domain 4 only loses smaller clusters during shearing and only begins to break up into
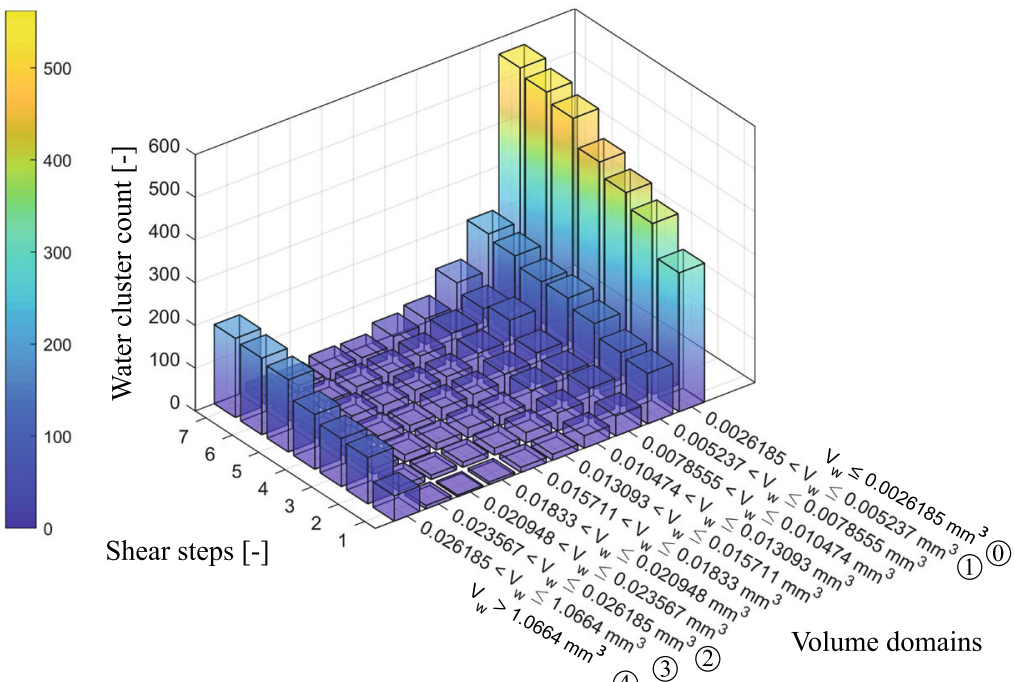

(4)
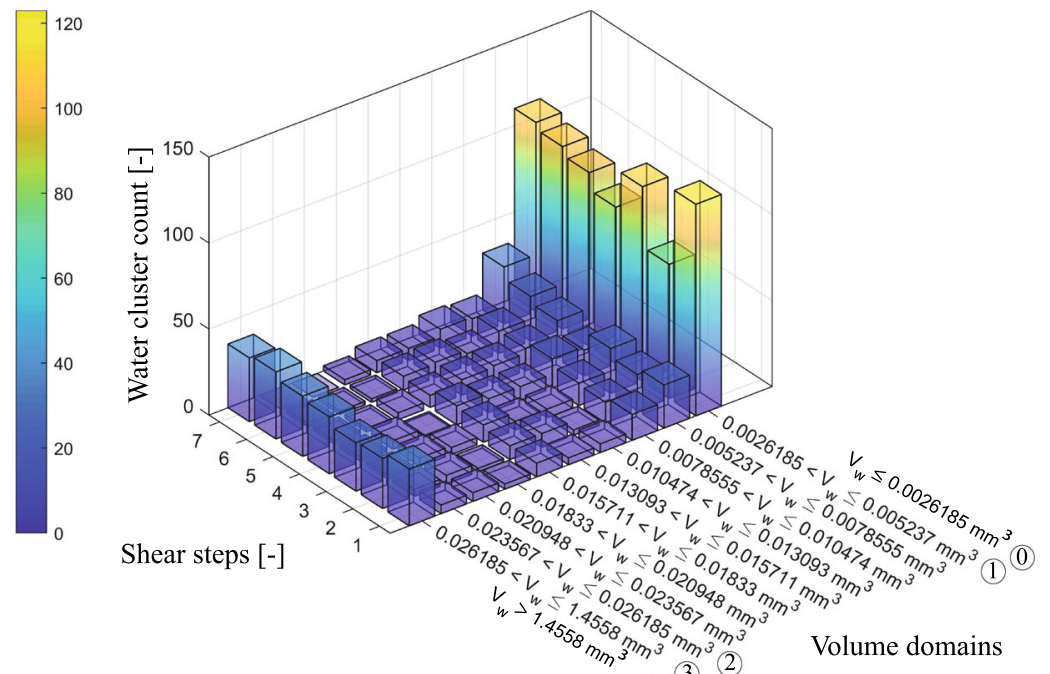

(4) (3)
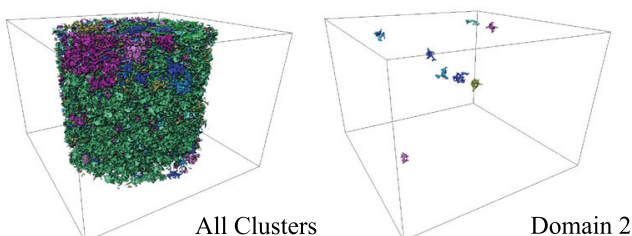

All Clusters
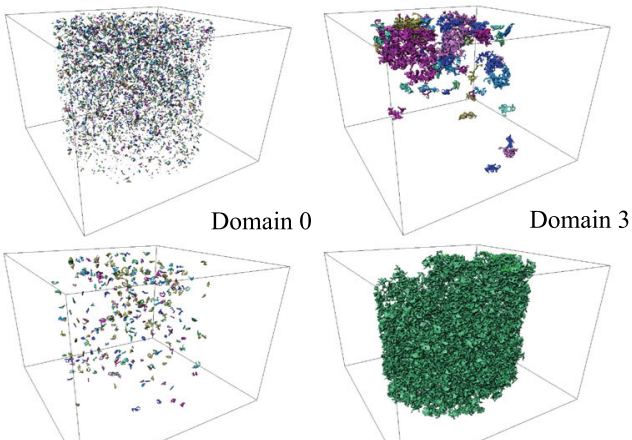

Domain 1
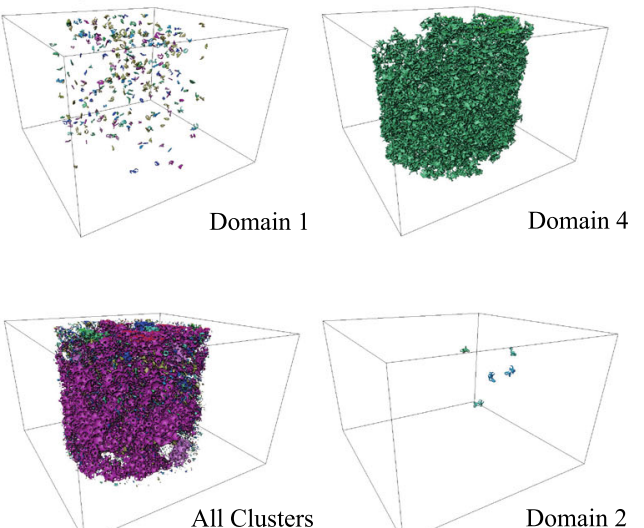

All Clusters

Domain 2
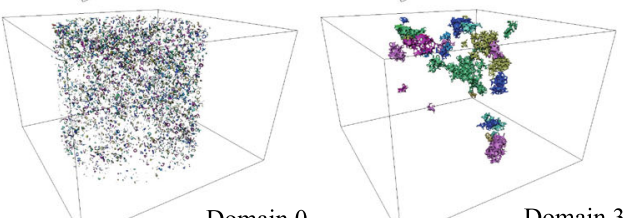

Domain 0
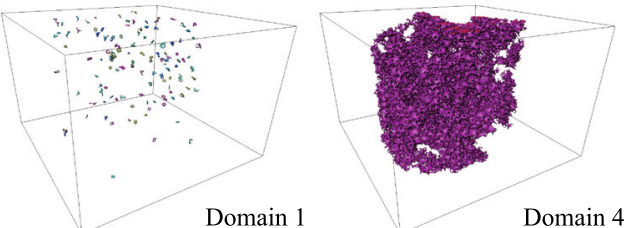

Fig. 13 Histogram of water cluster volumes during shearing of the driest sand specimen (top) and driest glass bead specimen (bottom). 3D renderings of labelled water clusters are presented for the first scan 
(a)
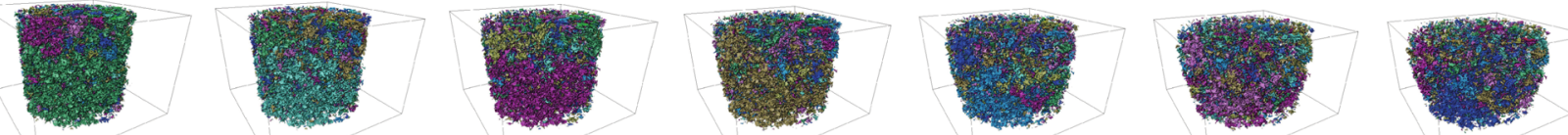

(b)
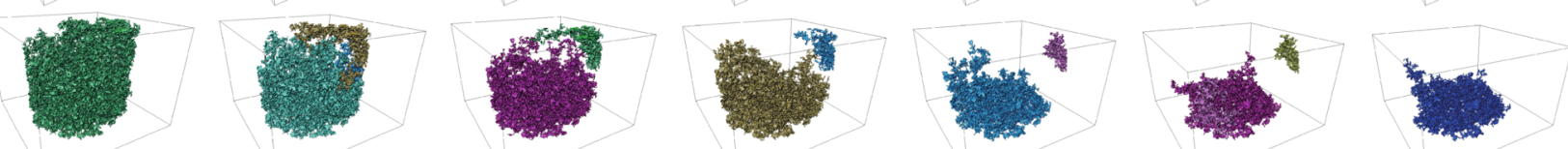

(c)
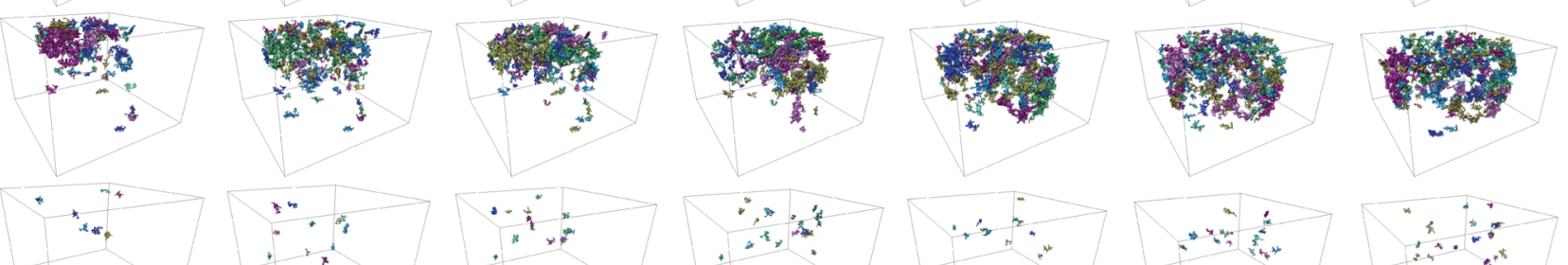

(d)
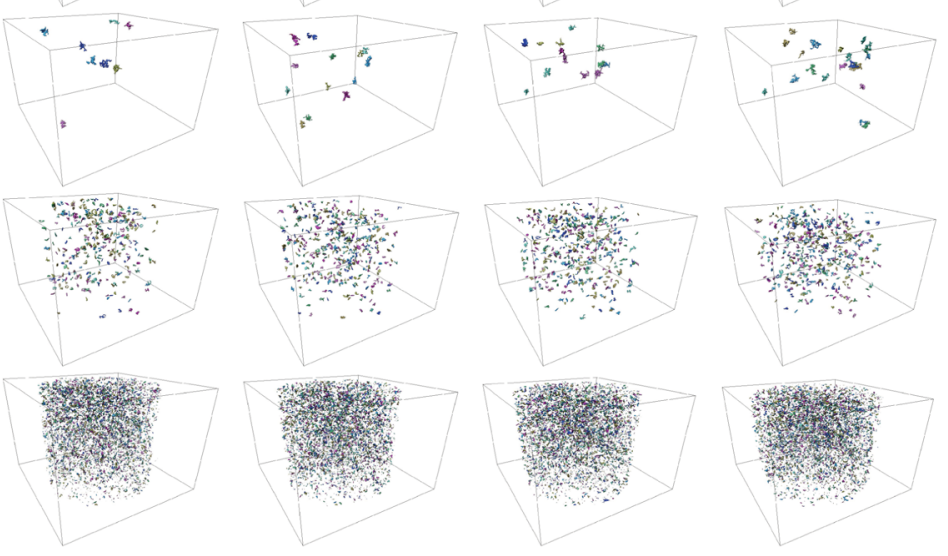

$\varepsilon=0.000$

$\varepsilon=0.025$

$\varepsilon=0.051$

$\varepsilon=0.076$
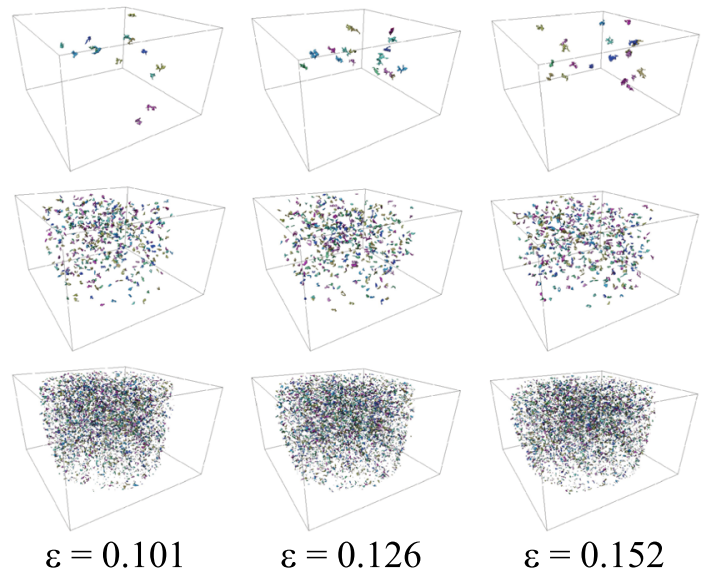

Fig. 14 Visualisation of independent water clusters during shearing of the driest sand specimen: a All clusters, b domain 4, c domain 3 , d domain 2, e domain 1, f domain 0. Compare to Fig. 13 (top)

larger clusters during the last two shear steps. In the sand specimen the breakup of the largest cluster into larger subclusters was noticed to already begin in the first shear step, compare Figs. 14 and 15.

\subsubsection{Grain kinematics}

As mentioned in Sect. 2.6, strain is computed on (constant strain) tetrahedra derived from grain centres (weighted by grain radius) in the finite strains framework. A vertical slice through the middle of incremental deviatoric strain fields for the driest specimens of Hamburg Sand and glass beads is shown in Fig. 16. These strain maps clearly indicate that strain localisation develops early in the tests, and a friction cone with very limited strains is apparent at the bottom of both specimens. Planar shear bands are visible in both specimens, with a tighter band observed for the beads, consistent with the tighter bands observed with rounder grains in [2].

In addition to the strain calculation, the spam software is used to identify grain-to-grain contacts and compute coordination number over time for two selected experiments on Hamburg Sand and glass beads, based on the improved grain contact detection procedure as described in [41] and [42]. The evolution of the total number of grain contacts and mean coordination number as a function of axial strain is shown in Fig. 17.

Prior to shearing the sand specimen shows more grain contacts compared to the glass bead specimen. For both materials, the number of grain contacts decreases during shearing, without reaching a stable plateau; the total reduction in grain contacts is more than three times higher in the sand compared to the glass beads. This grain contact loss is consistent with the observed dilatancy of both materials, which is also more pronounced in the sand specimens.

The mean coordination number is higher in the glass bead specimen and decreases more slowly during shearing as compared to the sand specimen. The distribution of coordination number per particle for the initial and final CT scans, shown for both materials in Fig. 27 in the Appendix, confirms that the glass beads are skewed towards higher coordination numbers compared to the sand. For both materials, the peak of the histograms shifts towards lower 
(a)
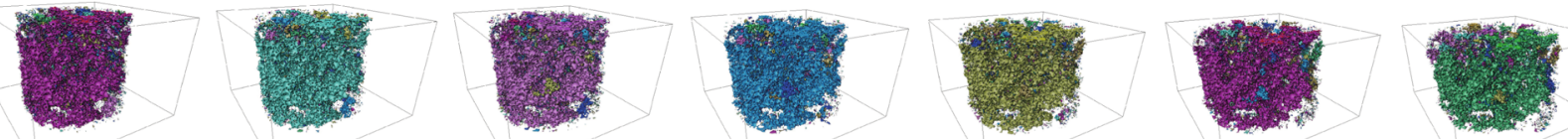

(b)
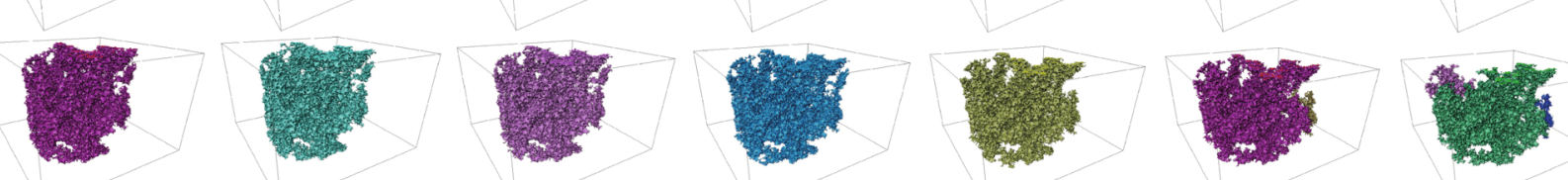

(c)
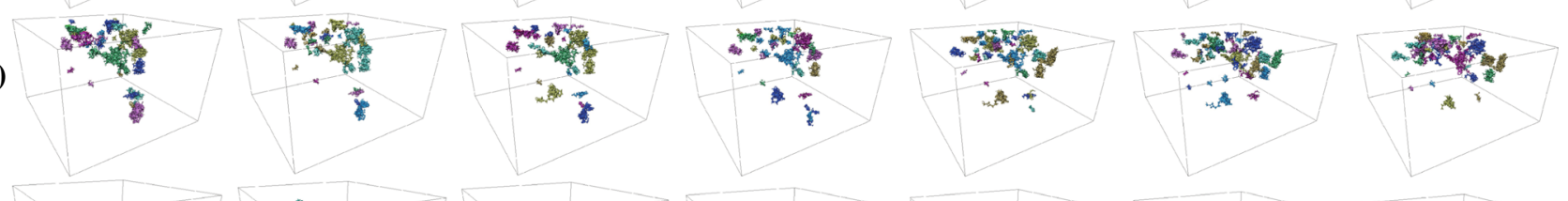

(d)

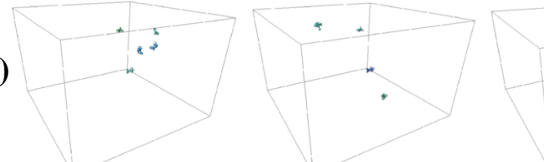

(e)

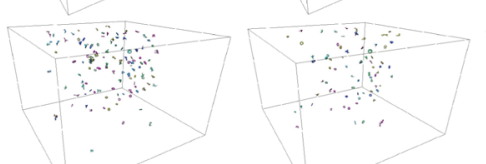

(f)
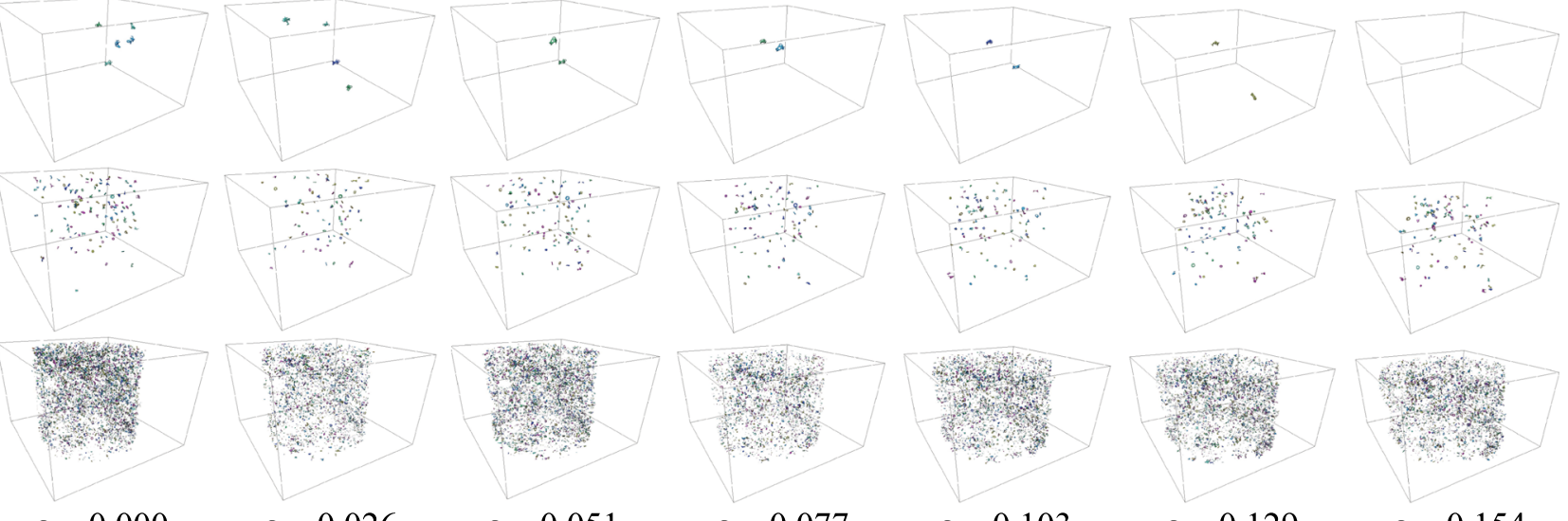

$\varepsilon=0.000$

$\varepsilon=0.026$

$\varepsilon=0.051$

$\varepsilon=0.077$

$\varepsilon=0.103$

$\varepsilon=0.129$

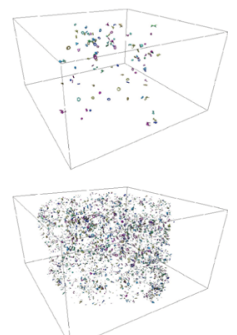

Fig. 15 Visualisation of independent water clusters during shearing of the driest glass bead specimen: a All clusters, $\mathbf{b}$ domain 4, $\mathbf{c}$ domain 3 , d domain 2, e domain 1, f domain 0. Compare to Fig. 13 (bottom)

coordination numbers by the end of the test; the contact loss is higher in the sand, with more grains with low coordination numbers occurring than in the glass beads. Note that similar results regarding the evolution of the coordination number have been obtained by [11] from triaxial compression tests on glass beads and sand specimens.

Figures 28 and 29 in the Appendix show, for both experiments discussed above, the spatial distribution of coordination number at the beginning and the end of the test. The grains are coloured by their value of coordination number in some slices. As expected, higher coordination numbers are found away from the edges of the sample.

\subsubsection{Local degree of saturation}

Since the tetrahedra described above link grain centres, they contain a pore whose volume and degree of saturation can be measured easily. Figure 18 shows a histogram of the degree of saturation of each pore (intended as each tetrahedron minus the solid phase) computed with the trinarised image for each of the scans of the driest specimens of Hamburg Sand (top) and glass beads (bottom).
This "bathtub" distribution reveals that pores are essentially fully saturated or mostly dry (although with some lower values of $S_{\mathrm{r}}$ also represented), supporting the approach in [10], where a binary degree of saturation is implicitly assumed in the pores. For both materials, as the samples are sheared the probability of pores with a low degree of saturation increases, whereas the probability of pores with high degree of saturation decreases. It is interesting to notice that the probability of pores with a high degree of saturation is higher in the glass beads compared to sand, which might be a result of the slightly higher macroscopic degree of saturation in the glass beads. Furthermore, the probability histograms for both materials look different for $S_{\mathrm{r}}<0.4$, which could be explained by the different pore size distributions of the two materials (although it must be remembered that the tessellation of non-spherical sand grains can create some pores with lessthan-ideal shapes in some cases).

\subsubsection{Linking strain and degree of saturation locally}

Since pores and strain are both defined on tetrahedra, it is therefore possible to link strains and pore evolution on this 


\section{Hamburg Sand (test 1)}

Step 1

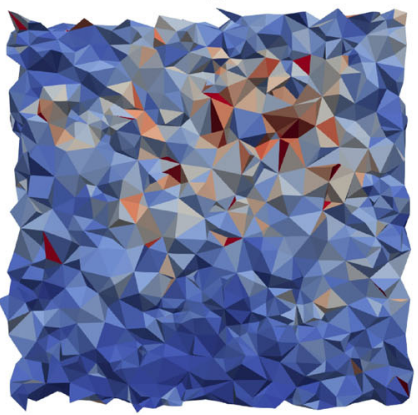

Step 4

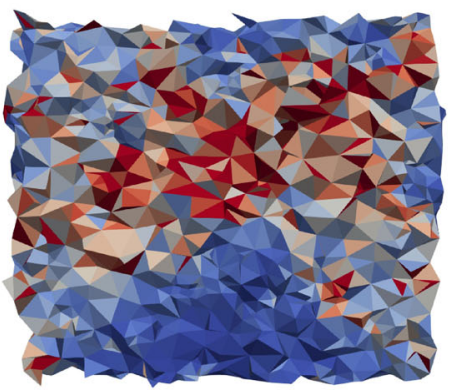

Step 2

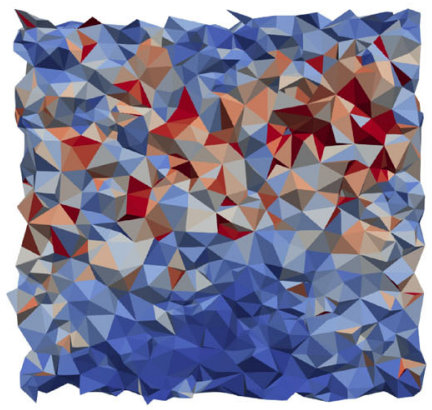

Step 5

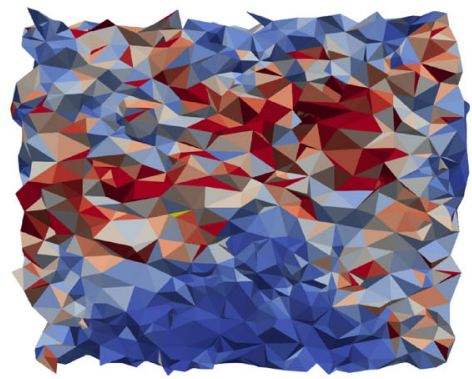

Step 3

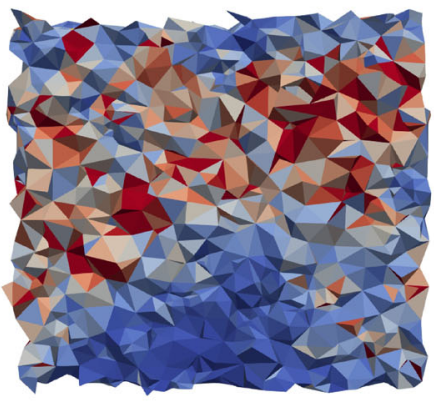

Step 6

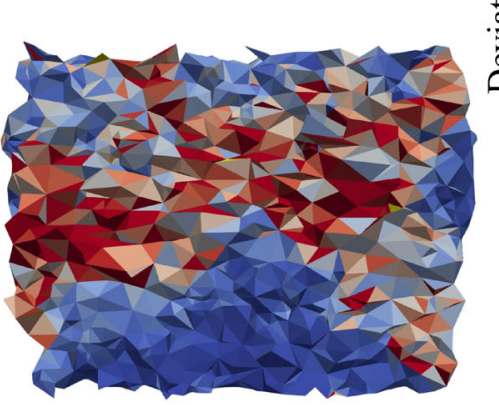

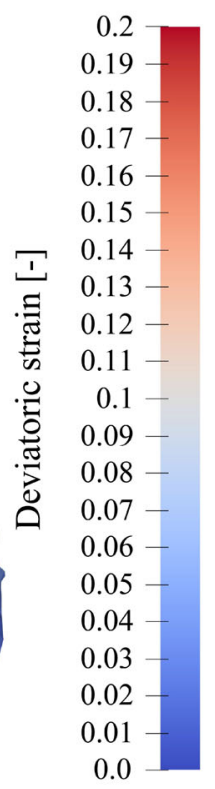

Glass beads (test 1)

Step 1

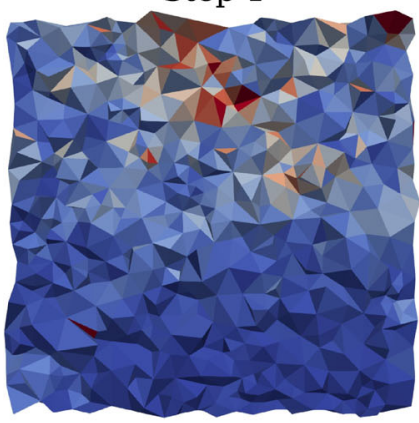

Step 4

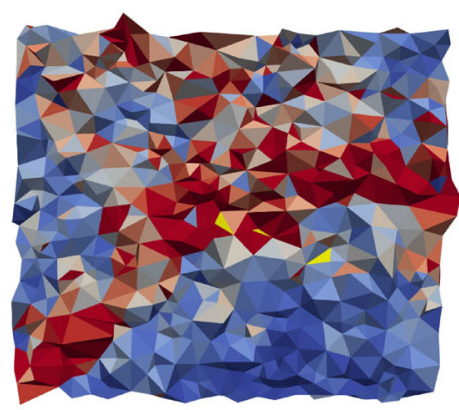

Step 2

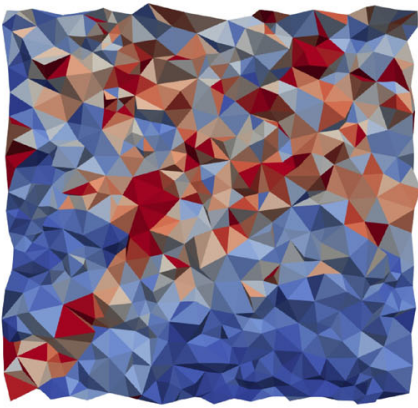

Step 5

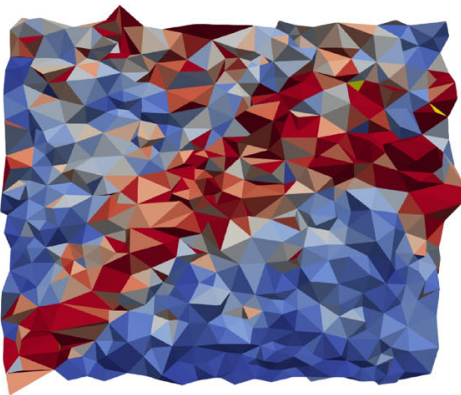

Step 3

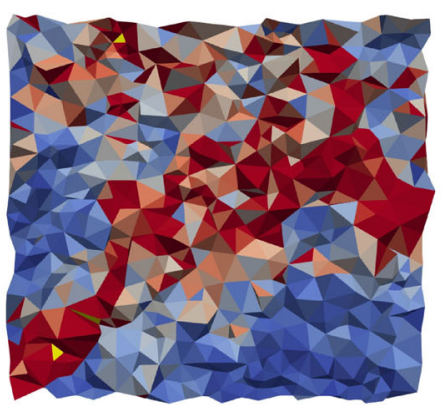

0.2

0.19

0.18

0.17

0.16

0.15

吉

0.13

0.12

0.11

Step 6

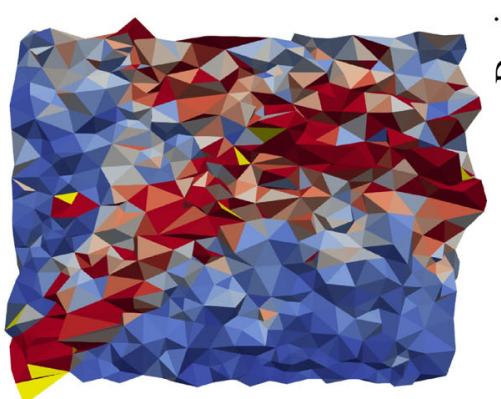

Fig. 16 Incremental deviatoric strain fields (computed in finite strains on tetrahedra and shown in x-direction using a "crinkle clip" to cut around them rather than through them) in the middle of the driest specimens of Hamburg Sand (top) and glass beads (bottom) 

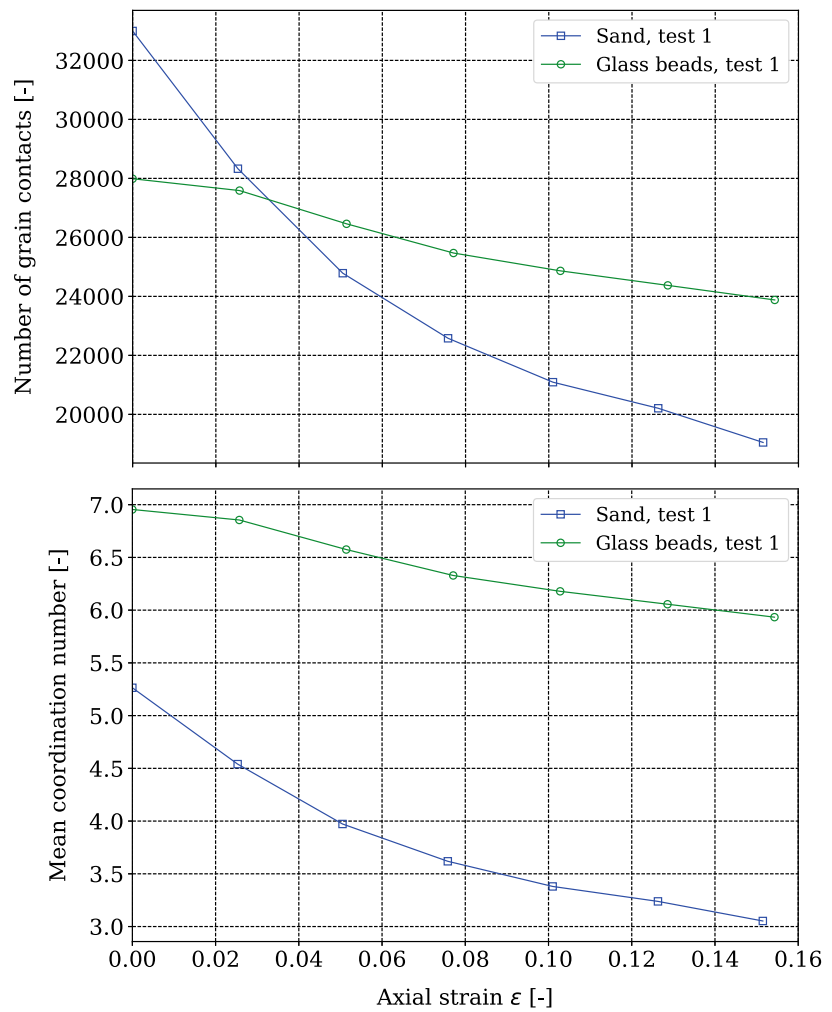

Fig. 17 Evolution of the number of grain contacts (top) and of coordination number (bottom) vs. axial strain for the driest specimen of Hamburg Sand and glass beads

same basis. For example, Figure 19 classifies each pore by degree of saturation (in the reference configuration, i.e., before the strain increment takes place) and for each class computes the volumetric and deviatoric strain (the median for each class, to avoid the effect of outliers) for each increment.

Each increment seems to indicate that the lower the degree of saturation, the higher the volumetric and deviatoric strain. Assuming that suction levels are relatively homogeneous throughout the specimens (minus a small vertical variation due to gravity), these results seem to confirm that where pores contain more water, the "reinforcing" effect of the suction, locally increasing shear strength and stiffness of the grain assembly, is stronger and thus lower volumetric as well as shear strains are observed. When the two materials are compared, higher (dilative) volumetric strain and lower deviatoric strain are measured in the sand. The higher dilation of sand specimens on the pore level confirms the macroscopic strain results shown in Fig. 8 and is likely linked to grain shape. The generally higher suction level in sand could furthermore explain the lower shear strains measured at the pore scale in this material. In both materials, especially for the higher degrees of saturation, the median values of both volumetric

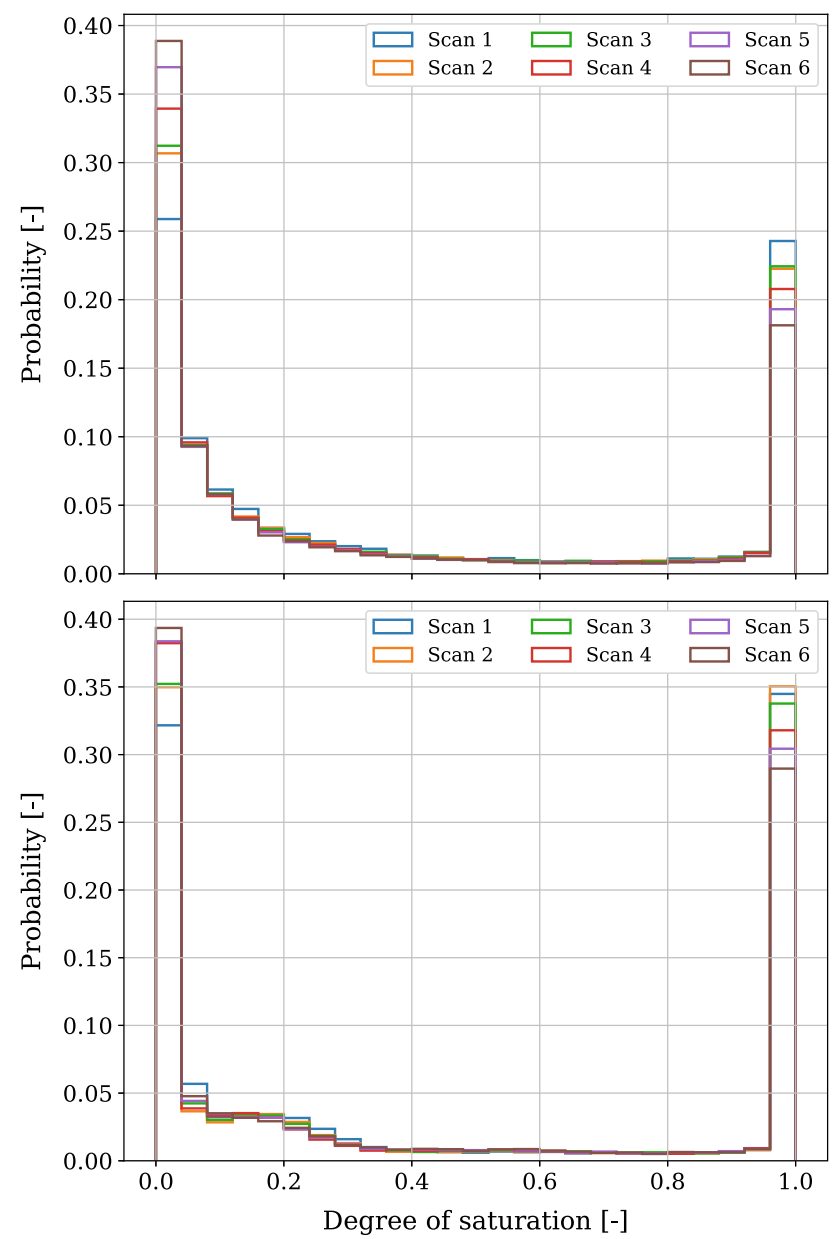

Fig. 18 Normalised histogram of degree of saturation for all pores in the first 6 scans for the driest specimen of Hamburg Sand (top) and glass beads (bottom)

and deviatoric strain increase in the first two to three shear steps and decrease afterwards. This decrease may be explained by the fact that there is increasingly strong strain localisation with shearing (see Fig. 16), so that the median values increasingly reflect the less-sheared part of the specimen.

\section{Summary and conclusion}

This paper has used in situ X-ray tomography to study partially saturated granular packings in uniaxial compression. Quantitative analysis of the acquired images allows, first of all, the macroscopic (force-displacement) response to be complemented by the sample volume evolution (dilative), which is precious information for the mechanical interpretation of the experiment that is difficult to obtain by other means for these materials. The shear strength of glass beads is much less than the sand-as expected-however, 

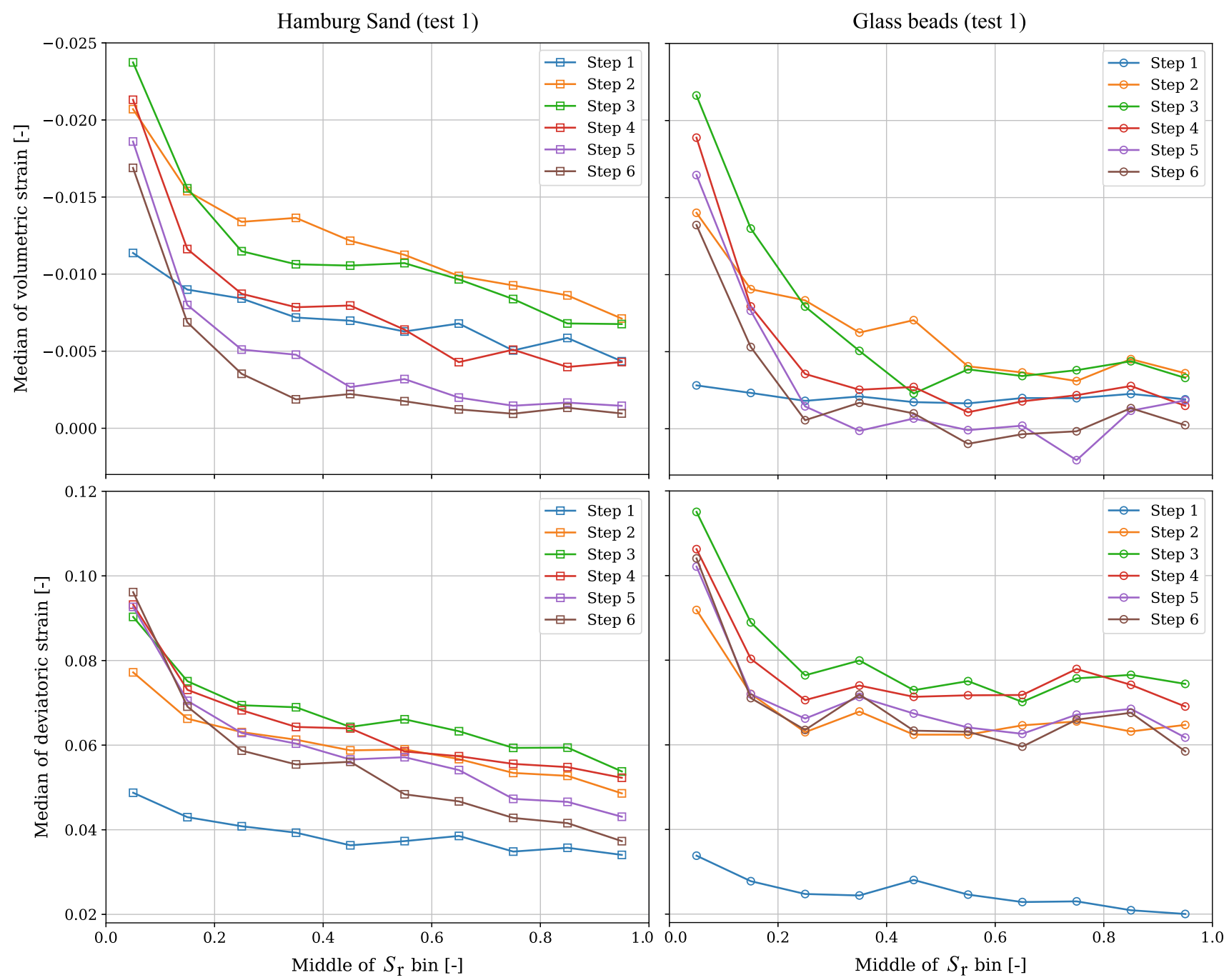

Fig. 19 Median of volumetric (negative = dilation) strain (top) and median of deviatoric strain (bottom) computed for pores classified by degree of saturation, presented for each shear step where a strain increment takes place of the driest specimens of Hamburg Sand (left column) and glass beads (right column)

unlike previous results the shear strength of each material does not seem to depend on the initial water content (which may be an artefact of starting and stopping loading for scanning). The volumetric strains of both materials during loading are clearly dependent on degree of saturation, with the drier samples dilating more.

The identification of solid, water and air phases in each 3D image allows a number of grain-scale quantities to be computed and studied both in space and during shearing. These initially allow the validation of the sample preparation procedure, both in terms of void ratio distribution (corresponding well to the classically measured one) and the distribution of water within specimens (corresponding less well and generally decreasing vertically). More advanced analysis allows the water's interfacial areas (to solid and air phases) to be computed and studied, the solid- water interfacial area essentially tracking the degree of saturation, but the air-water interfacial area showing a clear peak when plotted against degree of saturation, the peak is more pronounced (and at a lower degree of saturation) for sand. These quantities are key for micro-scale models of partially saturated granular materials as they are believed to represent essential contributors to effective stress in the unsaturated state. These results can be intuitively explained by considering the different pore morphologies and meniscus curvature in the two materials. However, a quantitative interpretation of these results would benefit from a measurement of suction during the experiment.

Individual enumeration of water clusters and particles allows further insight into the mechanisms underlying the shearing process: the evolution of the number and size of 
water clusters shows big differences between sand and glass bead samples (many more clusters in the sand), likely due to the differences in grain topology and thus pore topologies. Careful observation of the shape of the smaller water clusters confirms differences between the two materials. For the water clusters an irregular shaped network is noticed in sand specimens, whereas water clusters show regular repeated patterns in packings of glass beads ([32] describe these "liquid morphologies" inside packings of monodisperse glass beads as multiples of a single capillary bridge, named trimers, pentamers, tetrahedra and heptamers). The evolution of cluster sizes for both materials clearly shows that larger clusters tend to break into smaller ones under the joint effect of dilation and shearing.

Individually identified particles combined with particle tracking offer an ideal basis for the computation of a strain tensor on a Radical Delaunay triangulation, revealing regions of highly localised strain. Comparison of pore saturation and volumetric as well as deviatoric strain reveals a robust trend of higher saturation linked to lower volumetric and deviatoric strains.

The new and well-controlled data acquired during this experimental campaign are hopefully a useful contribution to the modelling efforts-regardless the scale-that can fully answer the research questions mentioned in the introduction. To this end they are made fully available to the community. The image data as well as uniaxial compression test data, analysed and discussed in this contribution, are hosted in the research data repository TORE at Hamburg University of Technology and can be downloaded from [29].

Technical improvements in the imaging speed would reduce evaporation as well as possible relaxation effects. This could be achieved by tolerating more noise or lower spatial resolution in lab X-ray scanners, or changing to synchrotron imaging (which would improve both temporal and spatial resolution). In situ suction measurements could also be a valuable addition to the measurements made during shearing. Finally, other mechanical tests, such as simple shear, direct shear or ring shear, represent interesting options for further research on the micro to macro shear behaviour of unsaturated granular media by means of in situ $\mathrm{CT}$ experiments.

\section{Appendix}

\section{Set-up for in situ experiments in the X-ray tomograph in Grenoble}

See Fig. 20.

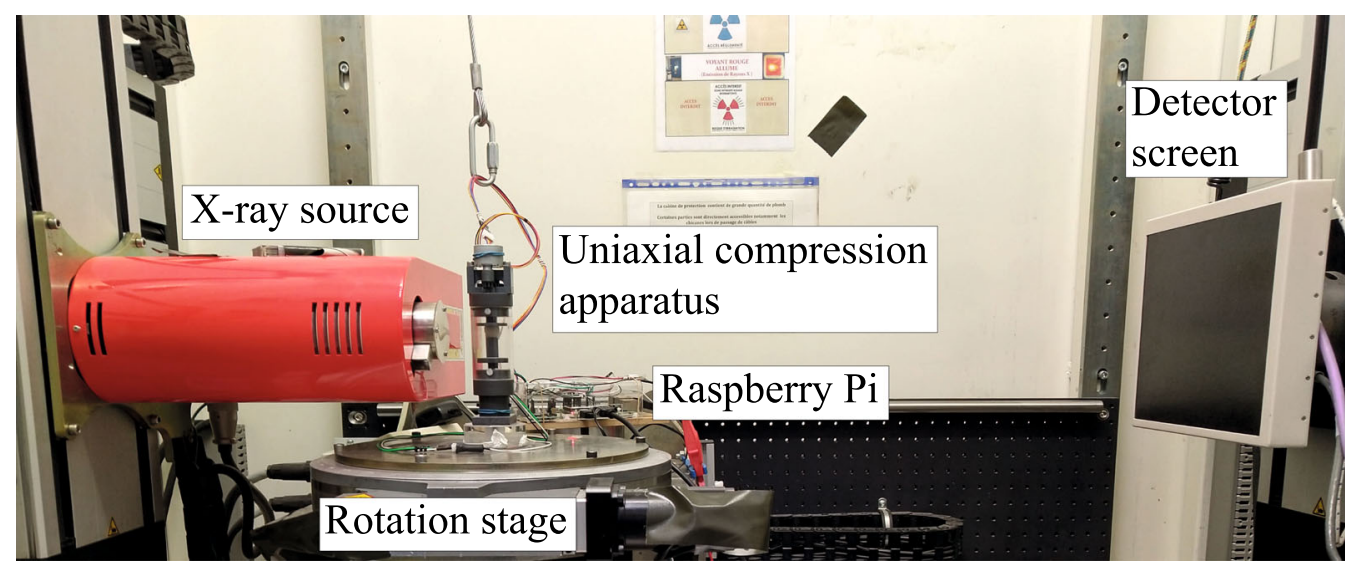

Fig. 20 Experimental set-up in the X-ray tomograph at Laboratoire 3SR, Université Grenoble Alpes 


\section{Specimen preparation for uniaxial compression \\ tests}

See Fig. 21.
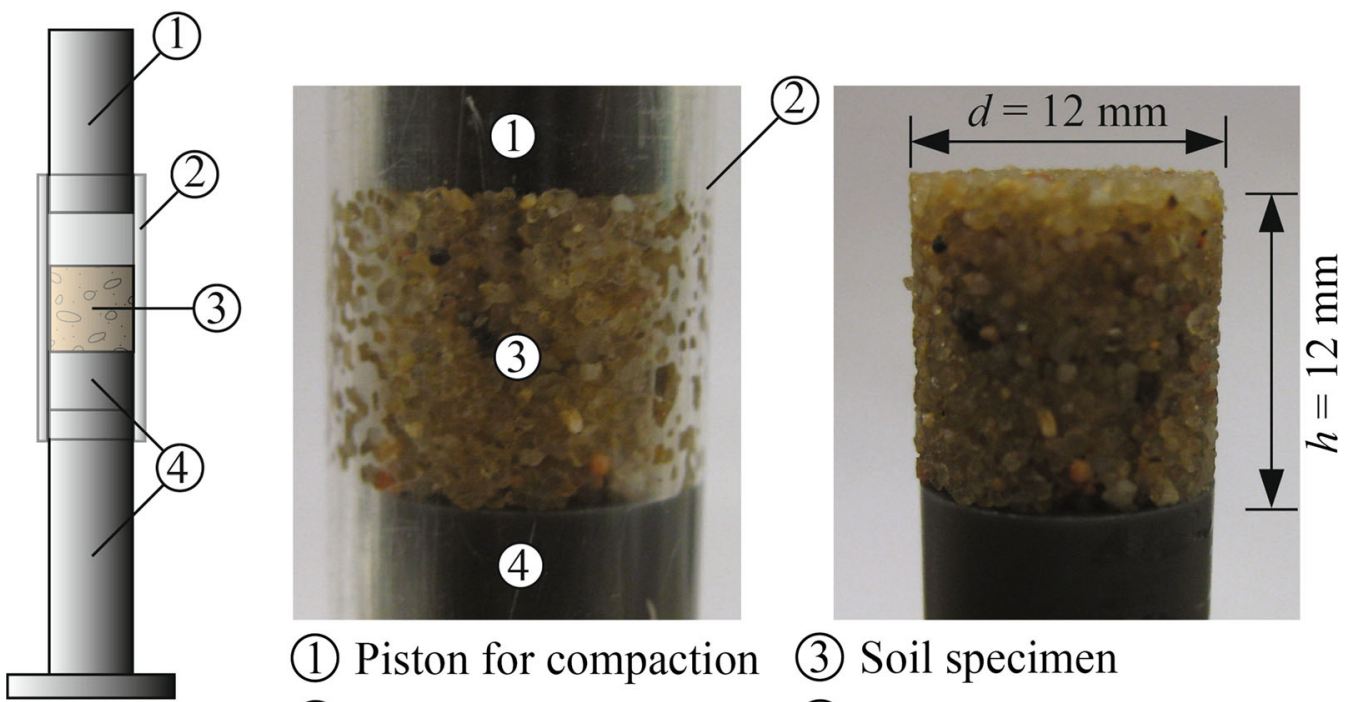
(1) Piston for compaction
(2) Acrylic tube

(3) Soil specimen

(4) Base plate and piston

Fig. 21 Principle of specimen preparation by compaction of unsaturated granular material in layers inside a hollow acrylic cylinder

\section{Measurement of specimen volume change from voxelised CT data}

See Fig. 22.

(a)

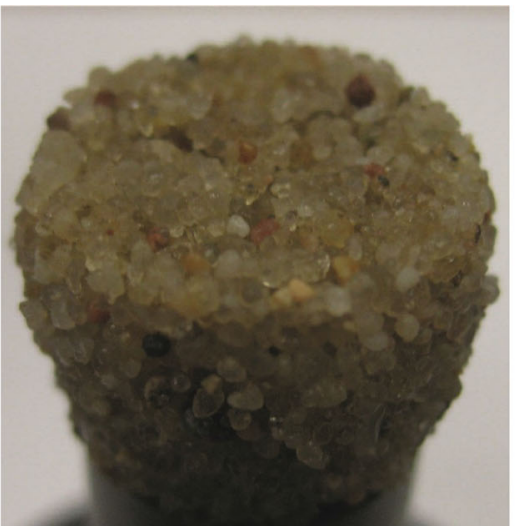

(b)

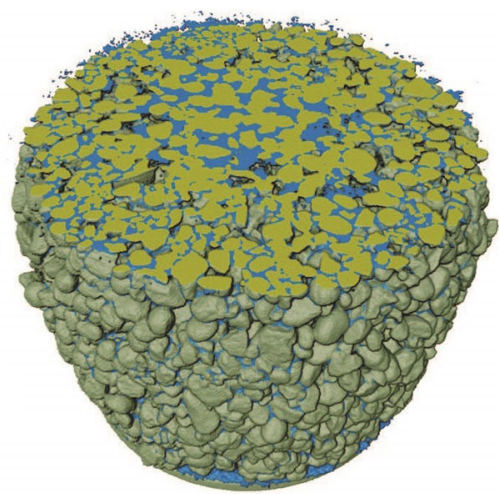

(c)

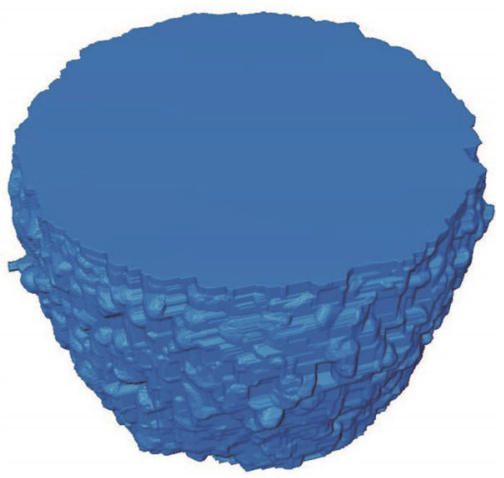

Fig. 22 Measurement of specimen volume change after shearing. a Sand specimen after 6 shear steps, b segmented 3D-volume consisting of sand (yellow) and water (blue), c approximated total specimen volume (color figure online) 


\section{Extraction of local microscopic specimen}

properties from segmented CT data

See Fig. 23.
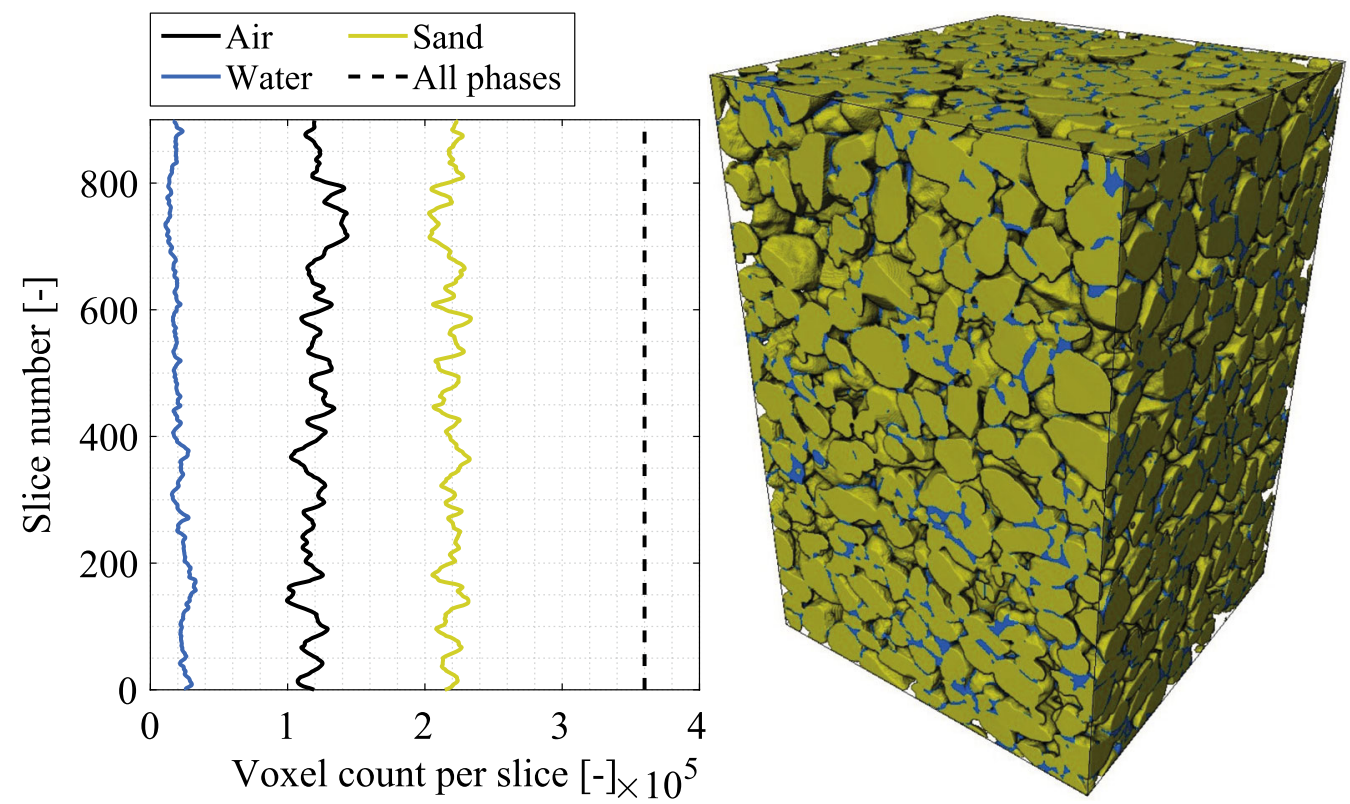

Fig. 23 Extraction of material phases per slice from segmented voxel data within a centred subvolume of $600 \times 600 \times 900 \mathrm{px}$ $(6.6 \times 6.6 \times 9.9 \mathrm{~mm})$ for the initial state of a sand specimen (test 1$)$ 


\section{Reconstructed and segmented 3D CT data showing macroscopic shear behaviour}

See Fig. 24.

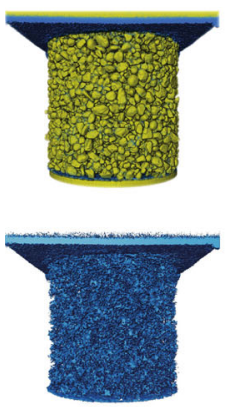

$\varepsilon=0.000$
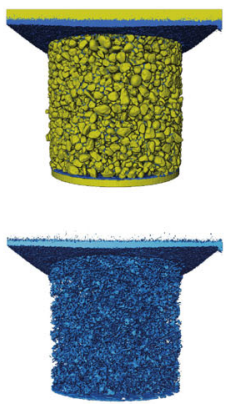

$\varepsilon=0.025$
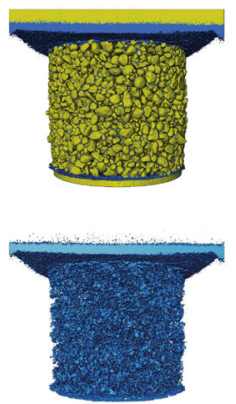

$\varepsilon=0.051$
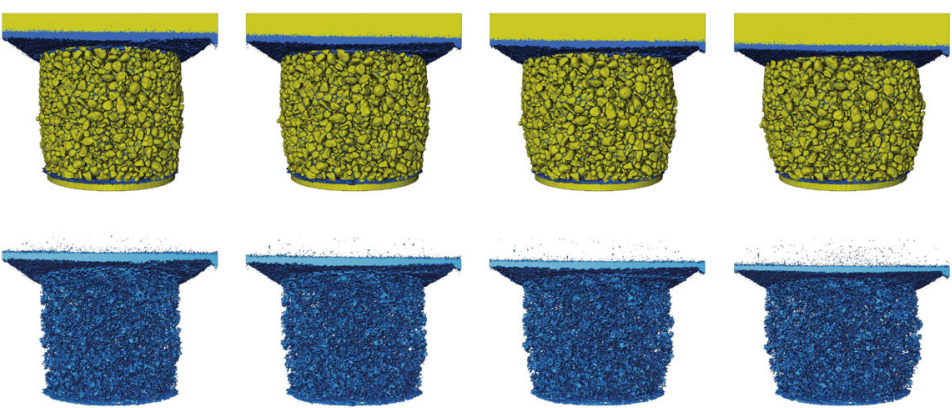

$\varepsilon=0.076$

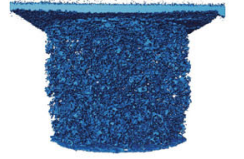

$\varepsilon=0.101$

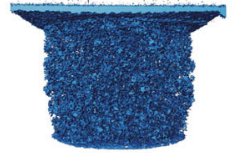

$\varepsilon=0.126$

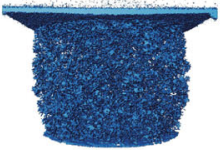

$\varepsilon=0.152$
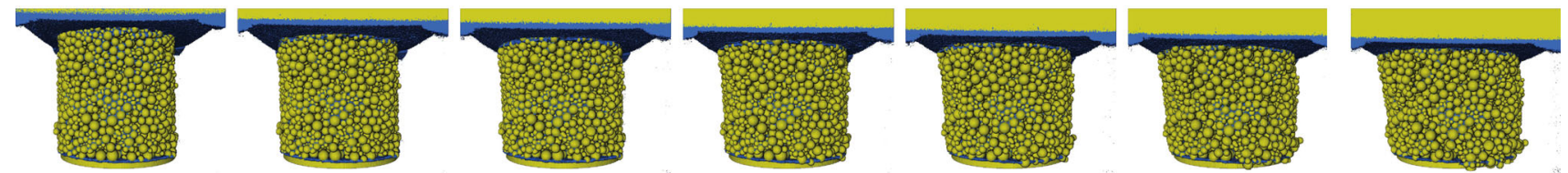

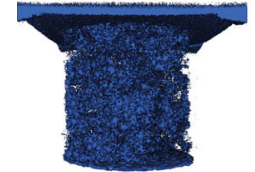

$\varepsilon=0.000$

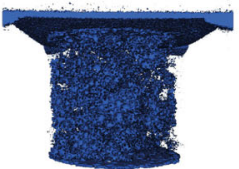

$\varepsilon=0.026$

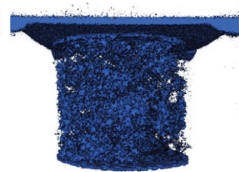

$\varepsilon=0.051$

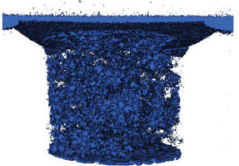

$\varepsilon=0.077$

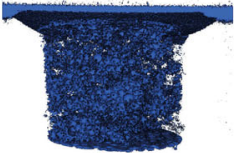

$\varepsilon=0.103$

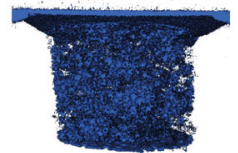

$\varepsilon=0.129$

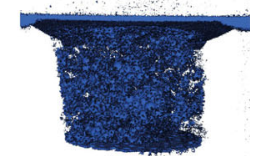

$\varepsilon=0.154$

Fig. 24 3D rendering of segmented volumes (non-air phases) acquired during uniaxial compression of sand specimen (test 1) and glass bead specimen (test 1). Not a single grain was noticed to fall out of the granular assemblies, kept together by visible water clusters and capillary bridges, during the experiment. However, movements of grain chains kept together by water bridges along the sides of the bottom loading plate could be noticed particularly for the glass beads. From the second shear step onwards, both specimens show bulging due to the strong dilatancy discussed above. When only the water phase is observed, a large deformation of the water clusters due to the dilatant behaviour of the grain skeleton can be noticed

\section{Further analysis of local void ratio and degree of saturation}

In order to observe the development of void ratio and degree of saturation locally in the specimen centre during shearing, these properties are calculated for another centred subvolume of $600 \times 600 \times 600 \mathrm{px}$. Being located in the specimen centre, this subvolume does not include boundary effects due to image noise and possible artefacts close to the top and bottom loading platens. The development of void ratio change $\Delta e$ as well as of degree of saturation vs. macroscopic axial strain is shown in Fig. 25.

The change of void ratio derived from the subvolume shows the same trends as the development of volumetric strain from Fig. 8. However, the local change of void ratio does not reproduce the initial contractant behaviour that is noticed on the macroscopic level. The tendency to dilative behaviour increases with decreasing initial degree of saturation. Due to the increasing pore volume, a nonlinear reduction in degree of saturation is noticed for all specimens during shearing. In order to investigate this effect without the influence of evaporation, the data have been corrected based on the measurement of specimen gravimetric water content and the corresponding trend lines shown in Fig. 5. Under the assumption that the evaporation is acting on all water cluster volumes in an equal way, the pore water volume loss can be compensated for to calculate a theoretical corrected degree of saturation and its change only due to pore volume change as shown in Fig. 26. The results indicate a reduction of degree of saturation inside the subvolume that is directly coupled to the increase in pore volume due to dilatancy. 

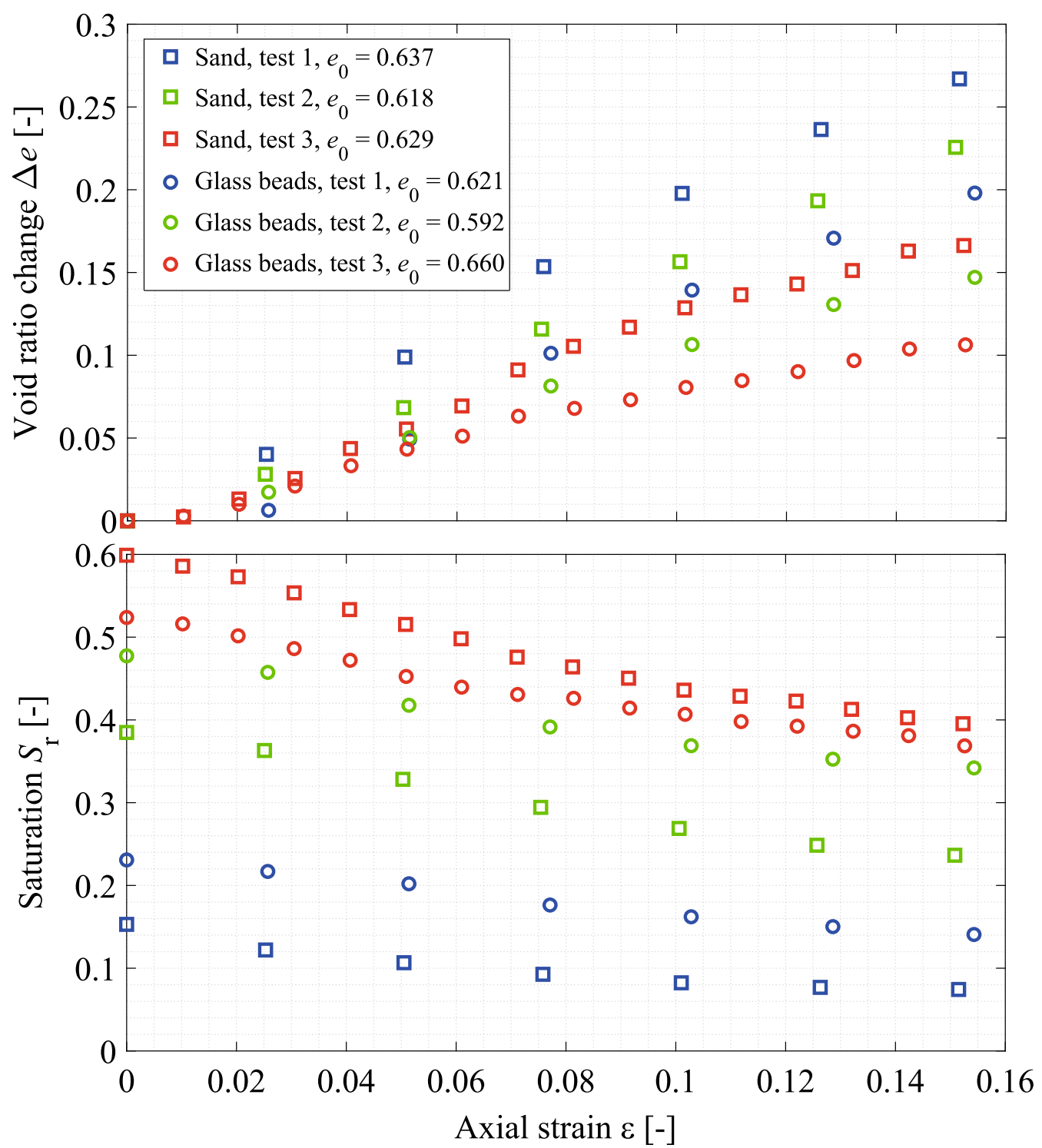

Fig. 25 Development of void ratio change (top) and degree of saturation (bottom) vs. axial strain within a centred subvolume of $600 \times 600 \times 600$ px during shearing 


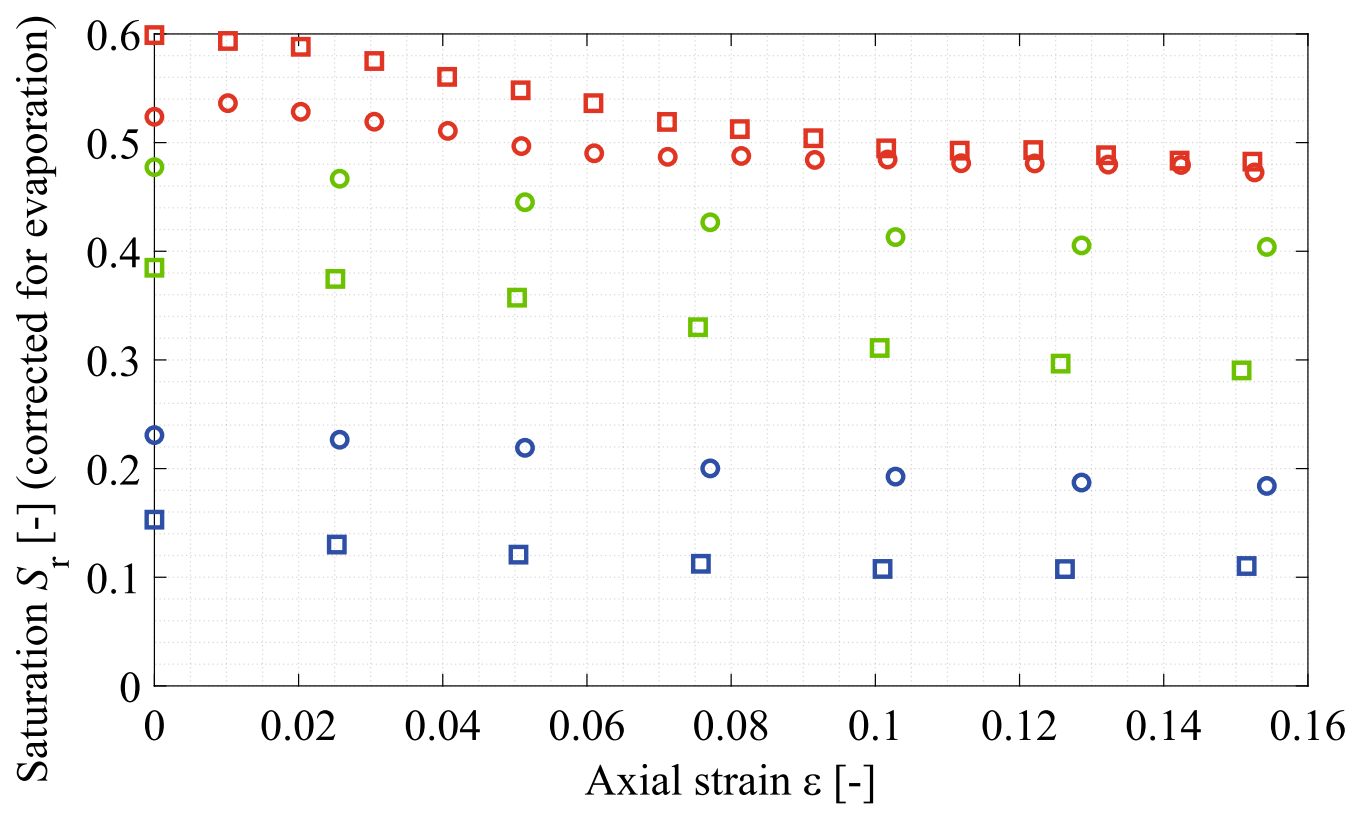

Fig. 26 Theoretical development of degree of saturation corrected for evaporation vs. axial strain within a centred subvolume of $600 \times 600 \times 600$ px during shearing, showing the isolated effect of dilatancy (same legend as in Fig. 25)

\section{Comparison of local and macroscopic initial void ratio and degree of saturation}

The local measurements of initial void ratio and initial degree of saturation from the subvolume show slight differences to these macroscopic soil properties, which are derived from initial macroscopic specimen volume and masses, such as water content from balancing. A comparison of macroscopic and local microscopic properties for the initial specimen state prior to shearing is given in Table 6.

Table 6 Comparison of initial macroscopic and local subvolumebased specimen properties

\begin{tabular}{ccccc}
\hline \multicolumn{6}{c}{ Hamburg Sand } \\
\hline $\begin{array}{c}\text { Test } \\
{[-]}\end{array}$ & $\begin{array}{c}e_{0, m} \\
{[-]}\end{array}$ & $\begin{array}{c}e_{0, s} \\
{[-]}\end{array}$ & $\begin{array}{c}S_{\mathrm{r} 0, \mathrm{~m}} \\
{[-]}\end{array}$ & $\begin{array}{c}S_{\mathrm{r} 0, \mathrm{~s}} \\
{[-]}\end{array}$ \\
\hline 1 & 0.6308 & 0.6373 & 0.2124 & 0.1530 \\
2 & 0.6383 & 0.6182 & 0.4153 & 0.3848 \\
3 & 0.6152 & 0.6292 & 0.6302 & 0.5990 \\
\hline Glass & beads & & & \\
\hline Test & $e_{0, m}$ & $e_{0, s}$ & $S_{\mathrm{r} 0, \mathrm{~m}}$ & $S_{\mathrm{r} 0, \mathrm{~s}}$ \\
{$[-]$} & {$[-]$} & {$[-]$} & {$[-]$} & {$[-]$} \\
\hline 1 & 0.5674 & 0.6211 & 0.3252 & 0.2308 \\
2 & 0.5673 & 0.5924 & 0.4601 & 0.4774 \\
3 & 0.5777 & 0.6602 & 0.5945 & 0.5238 \\
\hline
\end{tabular}

m: macroscopic, s: from subvolume
The absolute deviation of macroscopic and local void ratio, averaged for all three individual tests, is 0.014 for the sand compared to 0.054 for the glass beads. The same deviation of degree of saturation is 0.040 for the sand and 0.061 for the glass beads. The agreement of macroscopic and local specimen properties is better in the case of void ratio compared to degree of saturation. The deviations are generally lower for the sand compared to the glass beads. These differences might be due to more inhomogeneity of soil properties in the glass beads for which segregation effects as well as a higher gradient of the distribution of saturation are known to occur. Furthermore, the soil properties measured on a macroscopic level seem to be influenced by boundary effects close to the top and bottom loading plates as well as by a potential occurrence of shear zones in which dilatancy might be acting predominantly. 


\section{Further results of grain contact analysis}

See Figs. 27-29

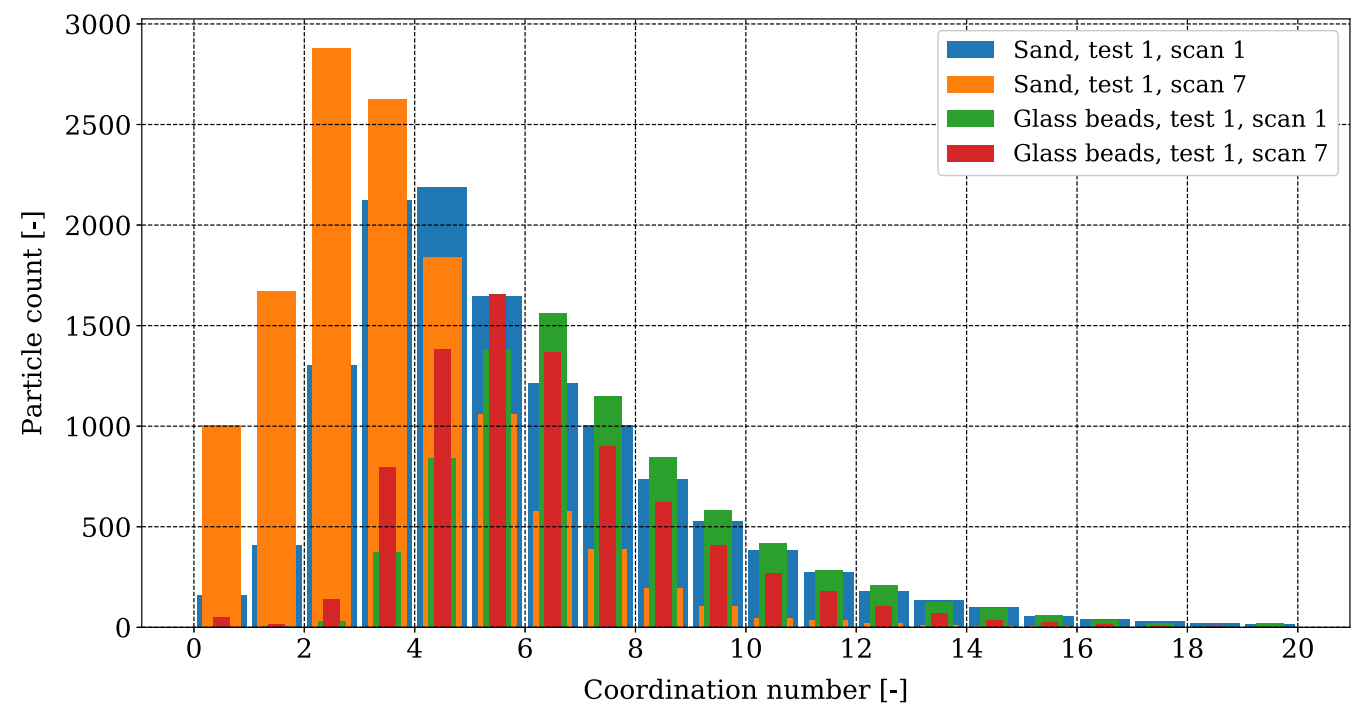

Fig. 27 Histogram-based comparison of the development of coordination numbers in a specimen of Hamburg Sand and glass beads (test 1) 

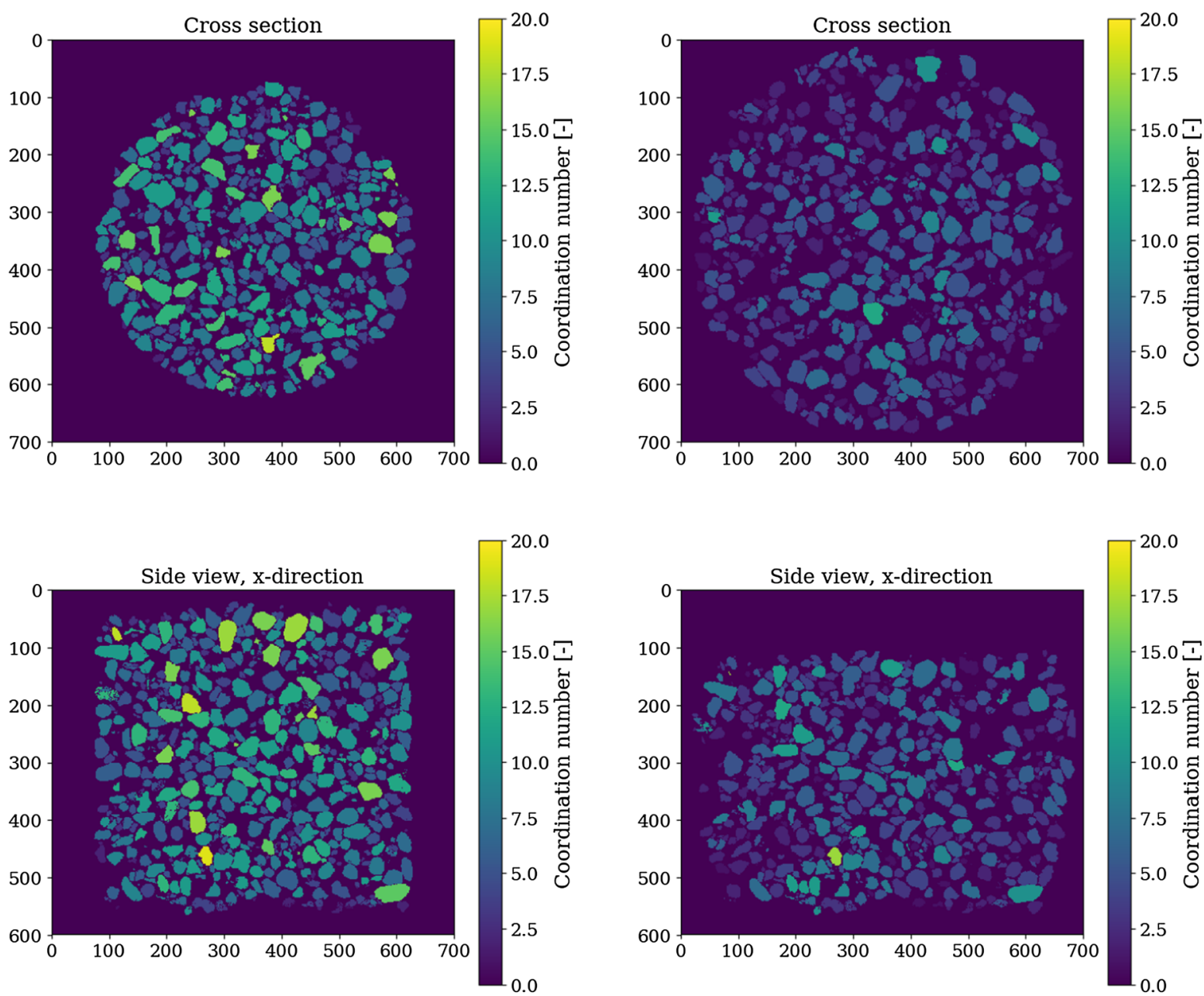

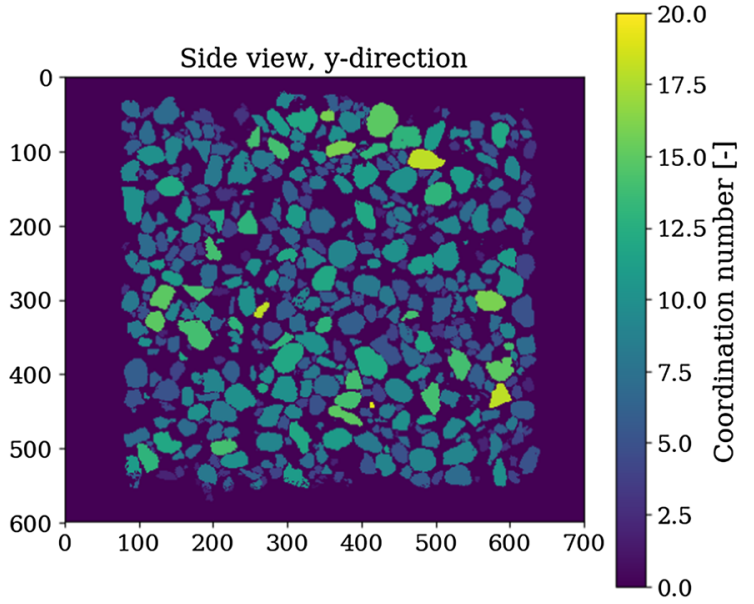

Sand, test 1 , scan 1

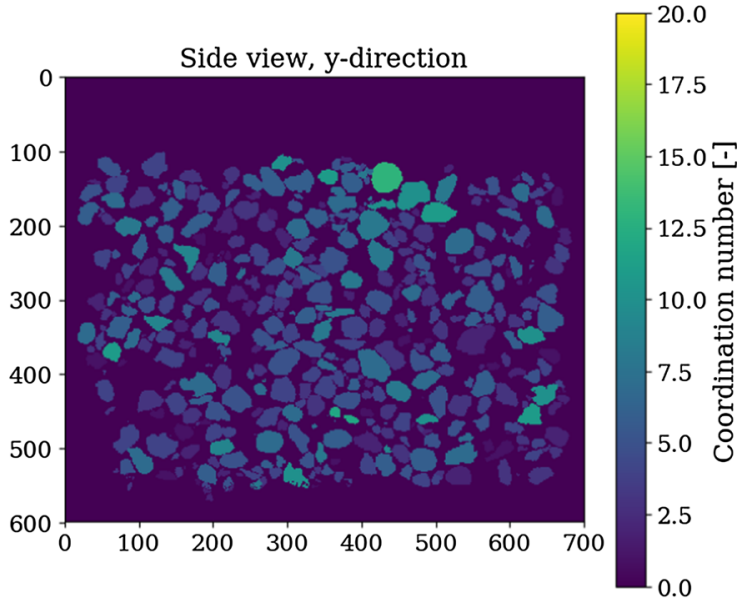

Sand, test $1, \operatorname{scan} 7$

Fig. 28 Visualisation of coordination numbers for every grain in a specimen of Hamburg Sand (test 1) in middle slices of the cross section and viewed from both sides 

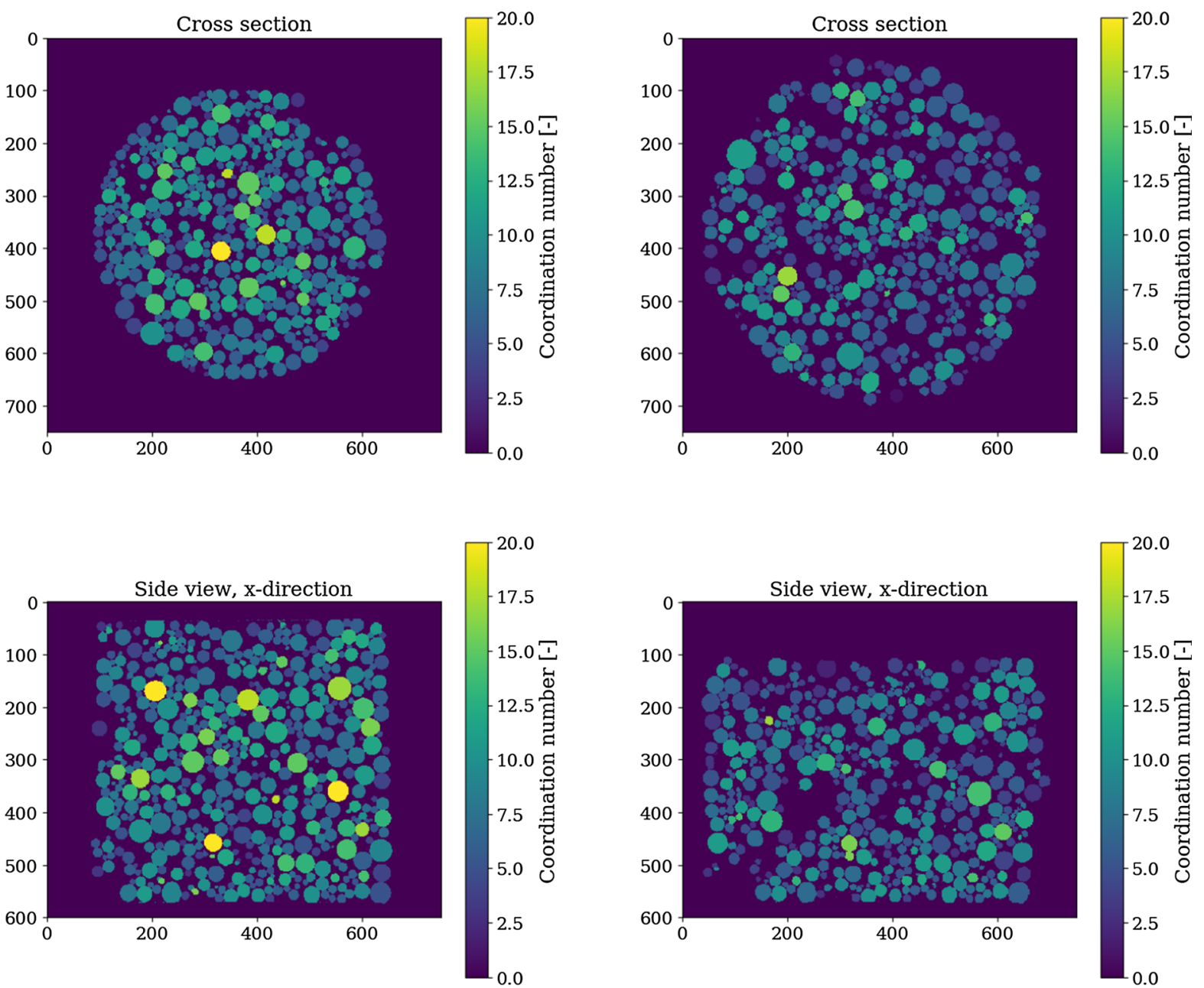

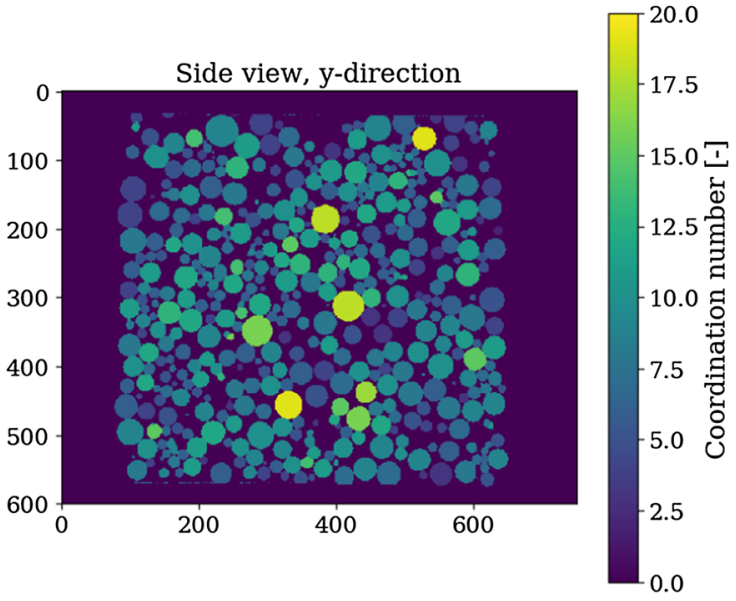

Glass beads, test 1 , scan 1

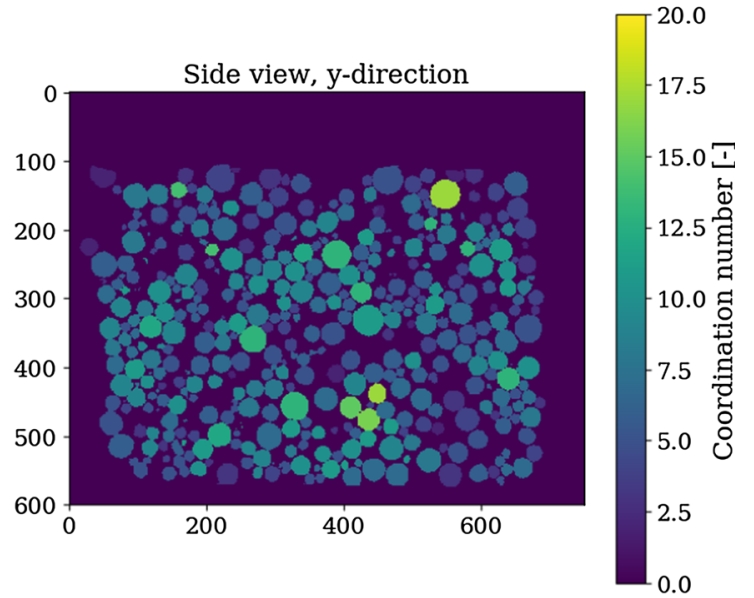

Glass beads, test 1 , scan 7

Fig. 29 Visualisation of coordination numbers for every grain in a specimen of glass beads (test 1) in middle slices of the cross section and viewed from both sides 
Acknowledgements The authors acknowledge the funding of this research by the German Research Foundation (Deutsche Forschungsgemeinschaft, DFG) in the framework of Research Training Group GRK 2462: Processes in natural and technical Particle-Fluid-Systems [13] at Hamburg University of Technology (TUHH). Laboratoire 3SR is part of the LabEx Tec 21 (Investissements d'Avenir grant agreement $n^{\circ}$ ANR-11-LABX-0030).

Funding Open Access funding enabled and organized by Projekt DEAL.

Open Access This article is licensed under a Creative Commons Attribution 4.0 International License, which permits use, sharing, adaptation, distribution and reproduction in any medium or format, as long as you give appropriate credit to the original author(s) and the source, provide a link to the Creative Commons licence, and indicate if changes were made. The images or other third party material in this article are included in the article's Creative Commons licence, unless indicated otherwise in a credit line to the material. If material is not included in the article's Creative Commons licence and your intended use is not permitted by statutory regulation or exceeds the permitted use, you will need to obtain permission directly from the copyright holder. To view a copy of this licence, visit http://creativecommons. org/licenses/by/4.0/.

\section{References}

1. Andò E (2013) Experimental investigation of microstructural changes in deforming granular media using x-ray tomography. $\mathrm{PhD}$ thesis, Mechanics [physics.med-ph]. Université de Grenoble

2. Andò E, Hall SA, Viggiani G, Desrues J, Bésuelle P (2012) Grain-scale experimental investigation of localised deformation in sand: a discrete particle tracking approach. Acta Geotech 7:1-13. https://doi.org/10.1007/s11440-011-0151-6

3. Andò E, Viggiani G, Hall S, Desrues J (2013) Experimental micro-mechanics of granular media studied by $\mathrm{x}$-ray tomography: recent results and challenges. Géotech Lett 3(3):142-146

4. Andrew M, Bijeljic B, Blunt MJ (2014) Pore-scale contact angle measurements at reservoir conditions using X-ray microtomography. Advances in Water Resources 68:24-31. https://doi.org/ 10.1016/j.advwatres.2014.02.014

5. Armstrong RT, Porter ML, Wildenschild D (2012) Linking porescale interfacial curvature to column-scale capillary pressure. Adv Water Resour 46:55-62. https://doi.org/10.1016/j.advwatres. 2012.05.009

6. Bagi K (1996) Stress and strain in granular assemblies. Mech Mate 22(3):165-177

7. Beare R, Lehmann G (2006) The watershed transform in itkdiscussion and new developments. Insight $\mathrm{J}$ 1:1-24

8. Bishop AW (1959) The principle of effective stress. Teknisk Ukeblad 106(39):859-863

9. Bruchon JF, Pereira JM, Vandamme M, Lenoir N, Delage P, Bornert M (2013) Full 3d investigation and characterisation of capillary collapse of a loose unsaturated sand using X-ray CT. Granular Matter 15:783-800

10. Chareyre B, Cortis A, Catalano E, Barthélemy E (2012) Porescale modeling of viscous flow and induced forces in dense sphere packings. Transport Porous Media 94(2):595-615

11. Cheng Z, Wang J (2018) Experimental investigation of interparticle contact evolution of sheared granular materials using X-ray micro-tomography. Soils Found 58:1492-1510. https://doi. org/10.1016/j.sandf.2018.08.008

12. Culligan KA, Wildenschild D, Christensen BSB, Gray WG, Rivers ML, Tompson AFB (2004) Interfacial area measurements for unsaturated flow through a porous medium. Water Resour Res. https://doi.org/10.1029/2004WR003278

13. DFG Research Training Group GRK 2462: Processes in natural and technical Particle-Fluid-Systems (PintPFS). https://gepris. dfg.de/gepris/projekt/390794421 ?language $=$ en (2019). Accessed 26 July 2021

14. Fredlund DG, Rahardjo H (1993) Soil mechanics for unsaturated soils. Wiley, Hoboken

15. Gastal ESL, Oliveira MM (2012) Adaptive manifolds for realtime high-dimensional filtering. ACM Trans Graph. https://doi. org/10.1145/2185520.2185529

16. Hall SA, Bornert M, Desrues J, Pannier Y, Lenoir N, Viggiani G, Bésuelle P (2010) Discrete and continuum analysis of localised deformation in sand using X-ray $\mu \mathrm{CT}$ and volumetric digital image correlation. Géotechnique 60(5):315-322

17. Higo Y, Morishita R, Kido R, Khaddour G, Salager S (2015) Local water-retention behaviour of sand during drying and wetting process observed by micro $\mathrm{x}$-ray tomography with trinarisation. Jpn Geotech Soc Spec Publ 2: 635-638 https://doi.org/10. 3208/jgssp.OTH-13

18. Higo Y, Oka F, Sato T, Matsushima Y, Kimoto S (2013) Investigation of localized deformation in partially saturated sand under triaxial compression using microfocus X-ray CT with digital image correlation. Soils Found 53:181-198

19. Jones AC, Arns CH, Sheppard AP, Hutmacher DW, Milthorpe BK, Knackstedt MA (2007) Assessment of bone ingrowth into porous biomaterials using micro-ct. Biomaterials 28:2491-250

20. Khaddour G (2015) Multi-scale characterisation of the hydromechanical behaviour of unsaturated sand: water retention and triaxial responses. Ph.d. thesis, Laboratoire 3SR, Université Grenoble Alpes

21. Khaddour G, Riedel I, Andò E, Charrier P, Bésuelle P, Desrues J, Viggiani G, Salager S (2018) Grain-scale characterization of water retention behaviour of sand using X-ray CT. Acta Geotech. https://doi.org/10.1007/s11440-018-0628-7

22. Kido R, Higo Y (2019) Distribution changes of grain contacts and menisci in shear band during triaxial compression test for unsaturated sand. In: Proc. of 7th Asia-Pacific Conference on Unsaturated Soils (AP-UNSAT 2019). https://www.jstage.jst.go. jp/article/jgssp/7/2/7_v07.096/_pdf/

23. Kido R, Higo Y (2020) Microscopic characteristics of partially saturated dense sand and their link to macroscopic responses under triaxial compression conditions. Acta Geotech. https://doi. org/10.1007/s11440-020-01049-w

24. Kido R, Higo Y, Takamura F, Morishita R., Khaddour G (2020) Morphological transitions for pore water and pore air during drying and wetting processes in partially saturated sand. Acta Geotech 15:1745-1761. https://doi.org/10.1007/s11440-02000939-3

25. Milatz M (2019) Zur Anwendung von Einplatinen-Computern in der bodenmechanischen Forschung und Lehre am Beispiel eines einaxialen Druckversuchs zur Untersuchung teilgesättigter granularer Böden (On the application of single-board computers in soil mechanical research and teaching using the example of an uniaxial compression test for the investigation of unsaturated granular soils). Geotechnik 42. https://doi.org/10.1002/gete. 201800015. (In German)

26. Milatz M (2020) Application of single-board computers in experimental research on unsaturated soils. In: R. Cardoso, C. Jommi, E. Romero (eds.) 4th European Conference on Unsaturated Soils (E-UNSAT 2020). Lisboa, Portugal. https://doi. org/10.1051/e3sconf/202019502022

27. Milatz M (2020) An automated testing device for continuous measurement of the hysteretic water retention curve of granular media. Acta Geotech. https://doi.org/10.1007/s11440-020-00922y 
28. Milatz M, Grabe J (2019) Microscopic investigation of the hydromechanical behavior of unsaturated granular media with X-ray CT. In: Proc. of 7th Asia-Pacific Conference on Unsaturated Soils (AP-UNSAT 2019). https://www.jstage.jst.go.jp/article/jgssp/7/2/ 7_v07.095/_pdf/

29. Milatz M, Hüsener N, Andò E, Viggiani G, Grabe J (2021) Data from in situ uniaxial compression experiments on unsaturated granular media with X-ray CT-imaging. TUHH Open Research (TORE), Hamburg University of Technology. https://doi.org/10. $15480 / 336.3291$

30. Moscariello M, Cuomo S, Salager S (2018) Capillary collapse of loose pyroclastic unsaturated sands characterized at grain scale. Acta Geotech 13:117-133

31. Peters A, Durner W (2008) Simplified evaporation method for determining soil hydraulic properties. J Hydrol 356:147-162

32. Scheel M, Seemann R, Brinkmann M, Di Michiel M, Sheppard A, Breidenbach B, Herminghaus S (2008) Morphological clues to wet granular pile stability. Nat Mater. https://doi.org/10.1038/ nmat 2117

33. Singh K, Jung M, Brinkmann M, Seemann R (2019) Capillarydominated fluid displacement in porous media. Annu Rev Fluid Mech 51:429-449. https://doi.org/10.1146/annurev-fluid-010518040342

34. Stamati O, Andò E, Roubin E, Cailletaud R, Wiebicke M, Pinzon G, Couture C, Hurley RC, Caulk R, Caillerie D, Matsushima T, Bésuelle P, Bertoni F, Arnaud T, Laborin AO, Rorato R, Sun Y, Tengattini A, Okubadejo O, Colliat JB, Saadatfar M, Garcia FE, Papazoglou C, Vego I, Brisard S, Dijkstra J, Birmpilis G (2020) 'spam': Software for practical analysis of materials. J Open Sour Softw 5(51):2286. https://doi.org/10.21105/joss.02286

35. UMS: HYPROP operation manual. http://library.metergroup. com/Manuals/UMS/Hyprop_Manual.pdf (2018). Accessed 29 Apr 2019

36. van Genuchten MT (1980) A closed-form equation for predicting the hydraulic conductivity of unsaturated soils. Soil Sci Soc Am J 44 (5):892-898 (1980)
37. Vanapalli SK, Nicotera MV, Sharma RS (2008) Axis translation and negative water column techniques for suction control. Geotech Geolo Eng 26(6):645-660

38. Viggiani G, Andò E, Takano E, Santamarina JC (2015) Laboratory $\mathrm{x}$-ray tomography: a valuable experimental tool for revealing processes in soils. Geotech Test J. https://doi.org/10.1520/ GTJ20140060

39. Wang J, Lambert P, De Kock T, Cnudde V, François B (2019) Investigation of the effect of specific interfacial area on strength of unsaturated granular materials by X-ray tomography. Acta Geotech 14:1545-1559. https://doi.org/10.1007/s11440-019-00765-2

40. Wiebicke M (2020) Experimental analysis of the evolution of fabric in granular soils upon monotonic loading and load reversals. PhD thesis, TU Dresden University

41. Wiebicke M, Andò E, Herle I, Viggiani G (2017) On the metrology of interparticle contacts in sand from x-ray tomography images. Measur Sci Technol. https://doi.org/10.1088/13616501/aa8dbf

42. Wiebicke M, Andò E, Šmilauer V, Herle I, Viggiani G (2019) A benchmark strategy for the experimental measurement of contact fabric. Granular Matter. https://doi.org/10.1007/s10035-019-0902-x

43. Wildenschild D, Hopmans JW, Vaz CMP, Rivers ML, Rikard D, Christensen BSB (2002) Using x-ray computed tomography in hydrology: systems, resolutions, and limitations. J Hydrol 267:285-297. https://doi.org/10.1016/S0022-1694(02)00157-9

44. Zhang B, Regueiro RA (2015) On large deformation granular strain measures for generating stress-strain relations based upon three-dimensional discrete element simulations. Int $\mathrm{J}$ Solids Struct 66:151-170

45. Zhou B, Ku Q, Wang H, Wang J (2020) Particle classification and intra-particle pore structure of carbonate sands. Eng Geol. https:// doi.org/10.1016/j.enggeo.2020.105889

Publisher's Note Springer Nature remains neutral with regard to jurisdictional claims in published maps and institutional affiliations. 